\title{
Update of the global electroweak fit and constraints on two-Higgs-doublet models
}

\author{
The Gfitter Group, J. Haller ${ }^{1}$, A. Hoecker ${ }^{2}$, R. Kogler ${ }^{1, a}$, K. Mönig ${ }^{3}$, T. Peiffer ${ }^{4}$, J. Stelzer ${ }^{2}$ \\ ${ }^{1}$ Institut für Experimentalphysik, Universität Hamburg, Hamburg, Germany \\ ${ }^{2}$ CERN, Geneva, Switzerland \\ ${ }^{3}$ DESY, Hamburg and Zeuthen, Germany \\ ${ }^{4}$ II. Physikalisches Institut, Georg-August-Universität Göttingen, Göttingen, Germany
}

Received: 7 March 2018 / Accepted: 2 August 2018 / Published online: 23 August 2018

(C) The Author(s) 2018

\begin{abstract}
We present an update of the global fit of the Standard Model electroweak sector to latest experimental results. We include new kinematic top quark and $W$ boson mass measurements from the LHC, a $\sin ^{2} \theta_{\text {eff }}^{\ell}$ result from the Tevatron, and a new evaluation of the hadronic contribution to $\alpha\left(M_{Z}^{2}\right)$. We present tests of the internal consistency of the electroweak Standard Model and updated numerical predictions of key observables. The electroweak data combined with measurements of the Higgs boson coupling strengths and flavour physics observables are used to constrain parameters of two-Higgs-doublet models.
\end{abstract}

\section{Contents}

1 Introduction . . . . . . . . . . . 1

2 Update of the global electroweak fit . . . . . . . 2

2.1 Input measurements and theoretical predictions 2 $W$ boson mass . . . . . . . . . . . 3 Top quark mass . . . . . . . . . . . . . 4 Theoretical calculations . . . . . . . . . 4

2.2 Results . . . . . . . . . . . . . . . . . . 4 Oblique parameters . . . . . . . . 8

3 Global fits in the two-Higgs-doublet model . . . . 8

3.1 Constraints from Higgs boson coupling mea-

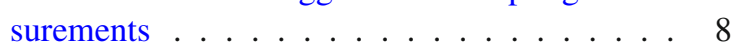

3.2 Constraints from flavour observables . . . . . 10 Experimental input data and theory calculation . 10 Results . . . . . . . . . . . . . 11

3.3 Constraints from the anomalous magnetic moment of the muon . . . . . . . . . . . 12

3.4 Constraints from electroweak precision data . . 13

3.5 Combined fit . . . . . . . . . . . 13

a e-mail: roman.kogler@physik.uni-hamburg.de

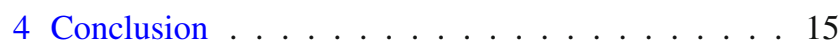

Appendix ................. 15

References............... 15

\section{Introduction}

Since the 1990'ies, electroweak precision data from LEP and SLD $[1,2]$ were used together with accurate Standard Model (SM) calculations to predict parameters of the theory. A first impressive confirmation of the predictive power of global fits in high-energy physics (HEP) was the discovery of the top quark at the Tevatron $[3,4]$ in 1995, with a mass in agreement with the predictions from global fits. Knowledge of the top quark mass $\left(m_{t}\right)$ made it possible to constrain the mass of the Higgs boson $\left(M_{H}\right)$. Increasing experimental and theoretical precision and the inclusion of constraints from direct Higgs boson searches from LEP and Tevatron narrowed the allowed mass range over time [5-9]. The discovery of the Higgs boson at the Large Hadron Collider (LHC) [10,11] with a mass around $125 \mathrm{GeV}$ impressively confirmed the SM at the quantum level. The historical development of the constraints is illustrated in Figs. 1 and 2, where the predictions of, respectively, $m_{t}$ and $M_{H}$ as derived from various global fits and direct measurements [3,4,10-21] are shown versus time.

With the measurement of $M_{H}$ the electroweak sector of the SM is overconstrained and the strength of global fits can be exploited to predict key observables such as the $W$ boson mass and the effective electroweak mixing angle, with a precision exceeding that of the direct measurements [22]. Since the last update of our fit [23] improved experimental results have become available that allow for more accurate tests of the internal consistency of the SM. Among these are the first determination of the $W$ boson mass at the LHC by the ATLAS 


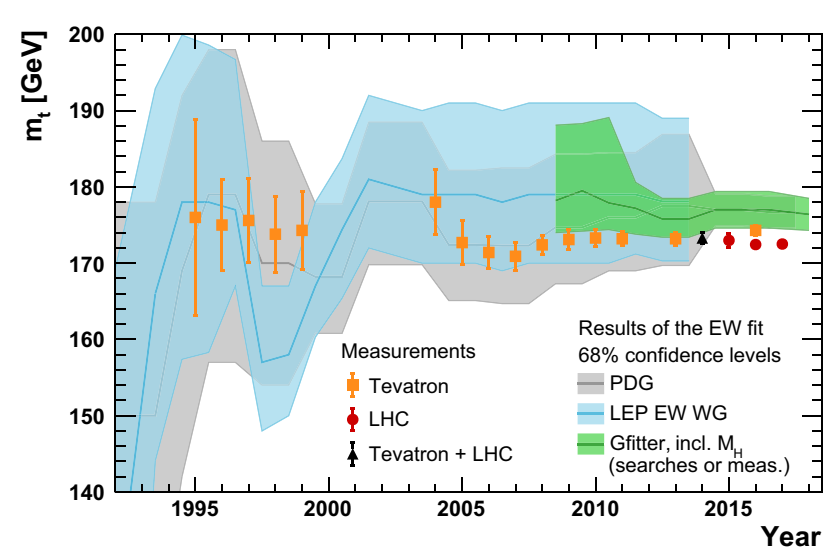

Fig. 1 Prediction of the top quark mass versus year as obtained by various analysis groups using electroweak precision data (grey [6], light blue [5], green [7]). The bands indicate the $68 \%$ confidence level. The direct $m_{t}$ measurements after the top quark discovery are displayed by the data points (orange $[3,4,12,16,17]$, red [14,15,21], black [13])

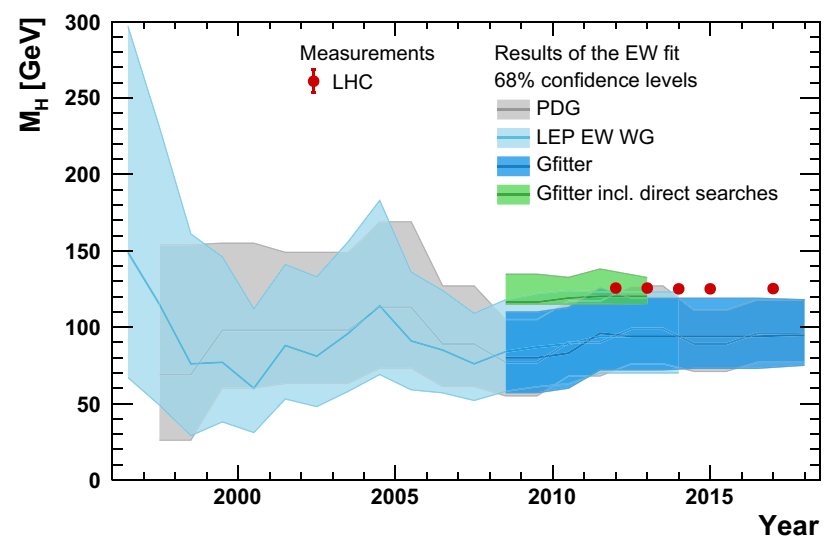

Fig. 2 Prediction of the Higgs boson mass versus year as obtained by various analysis groups using electroweak precision data (grey [6], light blue [5], dark blue [7]) and including direct search results (green [7]). The bands indicate the $68 \%$ confidence level. The direct $M_{H}$ measurements after the Higgs boson discovery are displayed by the red data points $[10,11,18-20]$

collaboration [24], new combined results of the top quark mass by the LHC experiments [15,21], a new combination of measurements of the effective leptonic electroweak mixing angles from the Tevatron experiments [25], a Higgs boson mass combination released by the ATLAS and CMS collaborations [18], and an updated value of the hadronic contribution to the running of the electromagnetic coupling strength at the $Z$ boson mass [26]. In the first part of this paper we present an update of the electroweak fit including these new experimental results and up-to-date theoretical predictions.

While the Higgs boson measurements so far agree with a minimal scalar sector as implemented in the SM, the question remains whether a more complex scalar sector may be realised in nature, possibly featuring a variety of Higgs boson states. Two-Higgs-doublet models (2HDM) [27] are a pop- ular SM extension in which an additional $S U(2)_{L} \times U(1)_{Y}$ scalar doublet field with hypercharge $Y=1$ is added to the SM leading to the existence of five physical Higgs boson states, $h, H, A, H^{+}$, and $H^{-}$, where the neutral $h$ may be identified with the discovered $125 \mathrm{GeV}$ Higgs boson as is assumed in this paper. The scalar $H$ boson has CP-even quantum number, $A$ is a CP-odd pseudo-scalar, and $H^{+}$and $H^{-}$carry opposite electric charge but have identical mass. No experimental hint for additional scalar states has been observed so far in direct searches [28-44]. In this situation global 2HDM fits, exploiting observables sensitive to these additional Higgs boson states via quantum corrections, can be used to constrain the allowed mass ranges and 2HDM mixing parameters. In the second part of this article such constraints are derived from a global fit using a combination of electroweak precision data, flavour physics observables, the anomalous magnetic moment of the muon, and measurements of the Higgs boson coupling strength to SM particles.

\section{Update of the global electroweak fit}

The updated global electroweak fit presented in this section uses the Gfitter framework. For a detailed discussion of the experimental data, the implementation of the theoretical predictions, and the statistical procedure employed by Gfitter we refer the reader to our previous publications $[9,22,23,45]$. A detailed list of all the observables, their values and uncertainties used in the fit, is given in the first two columns of Table 1. The description below discusses recent changes in the input quantities and calculations.

\subsection{Input measurements and theoretical predictions}

The electroweak precision data measured at the $Z$ pole and their correlations [1] as well as the width of the $W$ boson have not changed since our last analysis [23]. The update to the most recent world average values for the running $c$ and $b$ quark masses [46] has negligible impact on the fit result. This is also the case for the Run-1 LHC average of the Higgs boson mass, $M_{H}=125.09 \pm 0.21 \pm 0.11 \mathrm{GeV}$ [18], which we use now instead of a simple weighted average. ${ }^{1}$

New results are available for several observables with high sensitivity and potentially significant impact on the fit. We include new measurements of the $W$ boson and top quark masses as described in the following sections. For the first time we include as a separate fit input (assuming no correlation with other measurements) the latest combination of measurements of the effective leptonic electroweak

\footnotetext{
1 The Run-1 result on $M_{H}$ was confirmed by ATLAS and CMS measurements at $\sqrt{s}=13 \mathrm{GeV}[19,20]$.
} 
Table 1 Input values and fit results for the observables used in the global electroweak fit. The first and second columns list respectively the observables/parameters used in the fit, and their experimental values or phenomenological estimates (see text for references). The third column indicates whether a parameter is floating in the fit. The fourth column gives the results of the fit including all experimental data. In the

fifth column, the fit results are given without using the corresponding experimental or phenomenological estimate in the given row (indirect determination). The last column shows for illustration the result using the same fit setup as in the fifth column, but ignoring all theoretical uncertainties

\begin{tabular}{|c|c|c|c|c|c|}
\hline Parameter & Input value & Free in fit & Fit result & Fit w/o exp. input in line & Fit w/o exp. input in line, no theo. unc. \\
\hline$M_{H}[\mathrm{GeV}]$ & $125.1 \pm 0.2$ & Yes & $125.1 \pm 0.2$ & $90_{-18}^{+21}$ & $89_{-17}^{+20}$ \\
\hline$M_{W}[\mathrm{GeV}]$ & $80.379 \pm 0.013$ & - & $80.359 \pm 0.006$ & $80.354 \pm 0.007$ & $80.354 \pm 0.005$ \\
\hline$\Gamma_{W}[\mathrm{GeV}]$ & $2.085 \pm 0.042$ & - & $2.091 \pm 0.001$ & $2.091 \pm 0.001$ & $2.091 \pm 0.001$ \\
\hline$M_{Z}[\mathrm{GeV}]$ & $91.1875 \pm 0.0021$ & Yes & $91.1882 \pm 0.0020$ & $91.2013 \pm 0.0095$ & $91.2017 \pm 0.0089$ \\
\hline$\Gamma_{Z}[\mathrm{GeV}]$ & $2.4952 \pm 0.0023$ & - & $2.4947 \pm 0.0014$ & $2.4941 \pm 0.0016$ & $2.4940 \pm 0.0016$ \\
\hline$\sigma_{\text {had }}^{0}[\mathrm{nb}]$ & $41.540 \pm 0.037$ & - & $41.484 \pm 0.015$ & $41.475 \pm 0.016$ & $41.475 \pm 0.015$ \\
\hline$R_{\ell}^{0}$ & $20.767 \pm 0.025$ & - & $20.742 \pm 0.017$ & $20.721 \pm 0.026$ & $20.719 \pm 0.025$ \\
\hline$A_{\mathrm{FB}}^{0, \ell}$ & $0.0171 \pm 0.0010$ & - & $0.01620 \pm 0.0001$ & $0.01619 \pm 0.0001$ & $0.01619 \pm 0.0001$ \\
\hline$A_{\ell}(\star)$ & $0.1499 \pm 0.0018$ & - & $0.1470 \pm 0.0005$ & $0.1470 \pm 0.0005$ & $0.1469 \pm 0.0003$ \\
\hline $\sin ^{2} \theta_{\mathrm{eff}}^{\ell}\left(Q_{\mathrm{FB}}\right)$ & $0.2324 \pm 0.0012$ & - & $0.23153 \pm 0.00006$ & $0.23153 \pm 0.00006$ & $0.23153 \pm 0.00004$ \\
\hline $\sin ^{2} \theta_{\mathrm{eff}}^{\ell}$ (Tevt.) & $0.23148 \pm 0.00033$ & - & $0.23153 \pm 0.00006$ & $0.23153 \pm 0.00006$ & $0.23153 \pm 0.00004$ \\
\hline$A_{c}$ & $0.670 \pm 0.027$ & - & $0.6679 \pm 0.00021$ & $0.6679 \pm 0.00021$ & $0.6679 \pm 0.00014$ \\
\hline$A_{b}$ & $0.923 \pm 0.020$ & - & $0.93475 \pm 0.00004$ & $0.93475 \pm 0.00004$ & $0.93475 \pm 0.00002$ \\
\hline$A_{\mathrm{FB}}^{0, c}$ & $0.0707 \pm 0.0035$ & - & $0.0736 \pm 0.0003$ & $0.0736 \pm 0.0003$ & $0.0736 \pm 0.0002$ \\
\hline$A_{\mathrm{FB}}^{0, b}$ & $0.0992 \pm 0.0016$ & - & $0.1030 \pm 0.0003$ & $0.1032 \pm 0.0003$ & $0.1031 \pm 0.0002$ \\
\hline$R_{c}^{0}$ & $0.1721 \pm 0.0030$ & - & $0.17224 \pm 0.00008$ & $0.17224 \pm 0.00008$ & $0.17224 \pm 0.00006$ \\
\hline$R_{b}^{0}$ & $0.21629 \pm 0.00066$ & - & $0.21582 \pm 0.00011$ & $0.21581 \pm 0.00011$ & $0.21581 \pm 0.00004$ \\
\hline $\bar{m}_{c}[\mathrm{GeV}]$ & $1.27_{-0.11}^{+0.07}$ & Yes & $1.27_{-0.11}^{+0.07}$ & - & - \\
\hline $\bar{m}_{b}[\mathrm{GeV}]$ & $4.20_{-0.07}^{+0.17}$ & Yes & $4.20_{-0.07}^{+0.17}$ & - & - \\
\hline$m_{t}[\mathrm{GeV}]^{(\nabla)}$ & $172.47 \pm 0.68$ & Yes & $172.83 \pm 0.65$ & $176.4 \pm 2.1$ & $176.4 \pm 2.0$ \\
\hline$\Delta \alpha_{\text {had }}^{(5)}\left(M_{Z}^{2}\right)^{(\dagger \Delta)}$ & $2760 \pm 9$ & Yes & $2758 \pm 9$ & $2716 \pm 39$ & $2715 \pm 37$ \\
\hline$\alpha_{S}\left(M_{Z}^{2}\right)$ & - & Yes & $0.1194 \pm 0.0029$ & $0.1194 \pm 0.0029$ & $0.1194 \pm 0.0028$ \\
\hline
\end{tabular}

${ }^{(\star)}$ Average of $\operatorname{LEP}\left(A_{\ell}=0.1465 \pm 0.0033\right)$ and $\operatorname{SLD}\left(A_{\ell}=0.1513 \pm 0.0021\right)$ measurements, used as two measurements in the fit. The fit without the LEP (SLD) measurement gives $A_{\ell}=0.1470 \pm 0.0005\left(A_{\ell}=0.1467 \pm 0.0005\right)$. ${ }^{(\nabla)}$ Combination of experimental $(0.46 \mathrm{GeV})$ and theory uncertainty $(0.5 \mathrm{GeV}){ }^{(\dagger)}$ In units of $10^{-5} \cdot{ }^{(\triangle)}$ Rescaled due to $\alpha_{s}$ dependency

mixing angle from the Tevatron experiments, ${ }^{2} \sin ^{2} \theta_{\mathrm{eff}}^{\ell}=$ $0.23148 \pm 0.00033$ [25], and we use an updated value for the five quark flavour hadronic contribution to the running of the electromagnetic coupling strength at $M_{Z}, \Delta \alpha_{\text {had }}^{(5)}\left(M_{Z}^{2}\right)=$ $(2760 \pm 9) \cdot 10^{-5}$ [26].

\section{W boson mass}

The ATLAS collaboration has recently released the first LHC measurement of the mass of the $W$ boson [24]. Analysing their $7 \mathrm{TeV}$ dataset ATLAS measures $M_{W}=80370 \pm 7_{\text {stat }} \pm$ $11_{\text {exp syst }} \pm 14_{\text {model }} \mathrm{MeV}$. We include this result in the fit by combining it with the Tevatron $\left(M_{W}=80387 \pm 16 \mathrm{MeV}\right.$

$\overline{2}$ The $\sin ^{2} \theta_{\text {eff }}^{\ell}$ measurements of ATLAS $\left(\sin ^{2} \theta_{\text {eff }}^{\ell}=0.2308 \pm 0.0012\right.$ [47]) and CMS $\left(\sin ^{2} \theta_{\text {eff }}^{\ell}=0.23101 \pm 0.00052\right.$ [48]) are not included in the fit because of their presently insufficient precision and unknown correlations.
[49]) and LEP combinations $\left(M_{W}=80376 \pm 25_{\text {stat }} \pm\right.$ $22_{\text {syst }} \mathrm{MeV}$ [50]) as follows.

Using information from Ref. [49] we estimate the composition of individual statistical, experimental systematic and modelling uncertainties in the combined Tevatron result by $\pm 8_{\text {stat }} \pm 8_{\text {exp syst }} \pm 12_{\text {model }} \mathrm{MeV}$. All statistical and experimental systematic uncertainties are assumed to be uncorrelated among the three input results (ATLAS, Tevatron, LEP) as is the modelling uncertainty from LEP. The impact of the unknown correlation among the modelling uncertainties affecting the ATLAS and Tevatron measurements has been studied by varying its value between zero and one. For a large range of correlations we observe a stable average of $M_{W}=80379 \pm 13 \mathrm{MeV}$, which we use in the fit. ${ }^{3}$

\footnotetext{
3 A central value of $80379 \mathrm{MeV}$ is obtained for all possible values of the model correlation, except for coefficients exceeding 0.9 for which
} 


\section{Top quark mass}

For lack of a recent $m_{t}$ world average, we attempt here for the purpose of the fit a conservative combination of the most precise kinematic $m_{t}$ measurements obtained at the LHC. We combine the $m_{t}$ averages from ATLAS (172.51 \pm $\left.0.27_{\text {stat }} \pm 0.42_{\text {syst }} \mathrm{GeV}\right)[21]$ and CMS (172.47 $\pm 0.13_{\text {stat }} \pm$ $0.47_{\text {syst }} \mathrm{GeV}$ ) [15], which are based on 7 and $8 \mathrm{TeV}$ data. These averages include results from the dilepton [52-54], lepton+jets [14,55] and fully hadronic [56] channels. Assuming the overlapping fraction of the systematic uncertainties to be fully correlated (which corresponds to a correlation coefficient of $72 \%$ between the two measurements) we obtain the combined value $m_{t}=172.47 \pm 0.46 \mathrm{GeV}$ ( $p$ value of 0.84 ), which we use as input in the fit.

The latest average from the D0 collaboration $m_{t}=$ $174.95 \pm 0.40_{\text {stat }} \pm 0.64_{\text {syst }} \mathrm{GeV}$ [17] is barely compatible with the aforementioned average of the LHC measurements. A combination of the D0 average with the LHC average would result in $p$-values between $5 \cdot 10^{-3}$ and $3 \cdot 10^{-5}$, depending on the assumed correlation between the systematic uncertainties. The result from the CDF collaboration, $m_{t}=173.16 \pm 0.57_{\text {stat }} \pm 0.74_{\text {syst }} \mathrm{GeV}$ [57], agrees with the LHC average, with $p$-values between 0.40 and 0.51 depending on the correlation.

As in our previous work [23] we assign an additional theoretical uncertainty of $0.5 \mathrm{GeV}$ to the value of $m_{t}$ from hadron collider measurements due to the ambiguity in the kinematic top quark mass definition [58-62], the colour structure of the fragmentation process $[63,64]$, and the perturbative relation between pole and $\overline{\mathrm{MS}}$ mass currently known to four-loop order [65-68].

\section{Theoretical calculations}

The theoretical higher-order calculations used in Gfitter have not changed since our last update [23], except for new bosonic two-loop corrections to the $Z b \bar{b}$ vertex [69].

For the effective weak mixing angle $\sin ^{2} \theta_{\text {eff }}^{f}$ we use the parametrisations provided in [69-71], which include full two-loop electroweak [70,71] and partial three-loop and four-loop QCD corrections [72-79]. For bottom quarks, the calculations from Refs. $[69,80]$ are used. The new bosonic two-loop corrections are numerically small. They shift the prediction of the forward-backward asymmetry for $b$ quarks $A_{\mathrm{FB}}^{0, b}$ by $1.3 \cdot 10^{-5}$, which is two orders of magnitude smaller

\section{Footnote 3 continued}

a value of $80380 \mathrm{MeV}$ is found. A combined uncertainty of $13 \mathrm{MeV}$ is obtained for correlations between 0.4 and 0.9 , while smaller and larger correlation values yield $12 \mathrm{MeV}$ and $14 \mathrm{MeV}$, respectively. These values have been consistently calculated using the Best Linear Unbiased Estimate (BLUE) [51] and the least-squares averaging implemented in Gfitter [9]. than the experimental uncertainty and thus does not alter the fit results. We use the parametrisation of the full two-loop result [81] for predicting the mass of the $W$ boson, where we also include four-loop QCD corrections [77-79]. Full fermionic two-loop corrections for the partial widths and branching ratios of the $Z$ boson and the hadronic peak cross section $\sigma_{\text {had }}^{0}$ are used [82-84]. The dominant contributions from final-state QED and QCD radiation are included in the calculations [85-90]. The width of the $W$ boson is known up to one electroweak loop order, where we use the parametrisation given in Ref. [91].

The size and treatment of theoretical uncertainties are unchanged with respect to our last analysis [23].

\subsection{Results}

The fit uses as input observables the quantities and values given in the left rows of Table 1 . The fit parameters are $M_{H}$, $M_{Z}, m_{c}, m_{b}, m_{t}, \Delta \alpha_{\mathrm{had}}^{(5)}\left(M_{Z}^{2}\right), \alpha_{S}$, as well as ten theoretical uncertainty (nuisance) parameters constrained by Gaussian functions (see Ref. [23] for more details).

The fit results in a minimum $\chi^{2}$ value of 18.6 for 15 degrees of freedom, corresponding to a $p$ value of 0.23 . The results of the full fit for each observable are given in the fourth column of Table 1, together with the uncertainties estimated from their $\Delta \chi^{2}=1$ profiles. The fifth column in Table 1 gives the results obtained without using the experimental measurement corresponding to that row in the fit (indirect determination of the observable). The last column in Table 1 corresponds to the fits of the previous column but ignoring all theoretical uncertainties [23].

The left-hand panel of Fig. 3 displays the pulls each given by the difference of the global fit result of an observable (fourth column of Table 1) and the corresponding input measurement (second column of Table 1) in units of the measurement uncertainty. The right-hand panel of Fig. 3 shows for each observable the difference between the global fit result (fourth column of Table 1) and the indirect determination (fifth column of Table 1), as well as the the difference between the input measurements (first column of Table 1) and the indirect determination. The differences are expressed in units of the total uncertainty obtained by adding in quadrature the corresponding uncertainties of the indirect determination and the input measurement. Comparing the results of the indirect determination, shown as blue bars and centred on zero, with the input measurements, shown as dots with error bars, illustrates the precision of the prediction with respect to that of the corresponding measurement. As in our previous fits, a tension is observed in the leptonic and hadronic asymmetry observables, which is largest in the forward-backward asymmetry of the $b$ quarks, $A_{\mathrm{FB}}^{0, b}$. The impact of the new Tevatron $\sin ^{2} \theta_{\text {eff }}^{\ell}$ measurement on the fit result is small due to yet insufficient precision. 


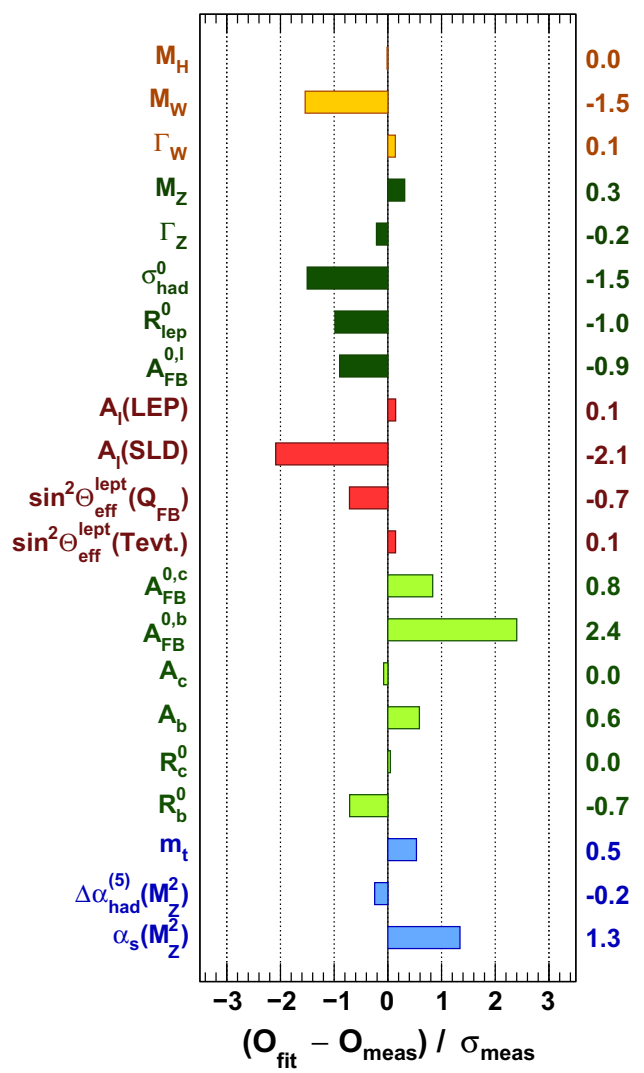

Fig. 3 Left: comparison of the fit results with the input measurements in units of the experimental uncertainties. Right: comparison of the fit results and the input measurements with the indirect determinations in units of the total uncertainties. Analog results for the indirect determina-

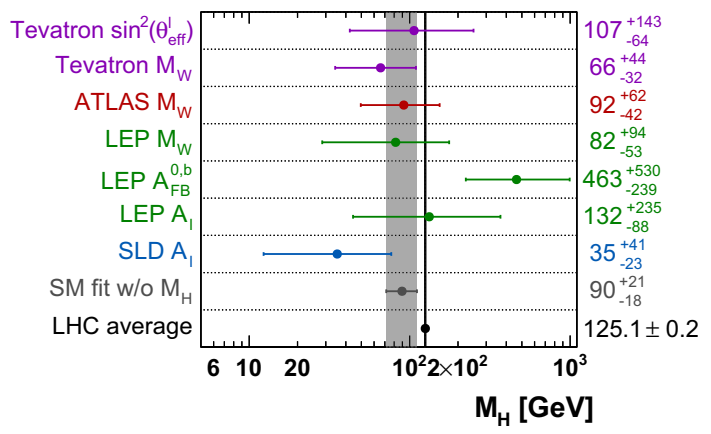

Fig. 4 Comparison of the constraints on $M_{H}$ obtained indirectly from individual observables with the fit result and the direct LHC measurement. For the indirect determinations among the four observables providing the strongest $M_{H}$ constraints (namely $\sin ^{2} \theta_{\mathrm{eff}}^{\ell}, M_{W}, A_{\mathrm{FB}}^{0, b}$ and $A_{\ell}$ ) only the one indicated in a given row of the plot is included in the fit. The results shown are not fully independent

Figure 4 displays the indirect determination of the Higgs boson mass from fits in which among the four observables providing the strongest $M_{H}$ constraints (namely $\sin ^{2} \theta_{\text {eff }}^{\ell}$, $M_{W}, A_{\mathrm{FB}}^{0, b}$ and $\left.A_{\ell}\right)$ only the one indicated in a given row

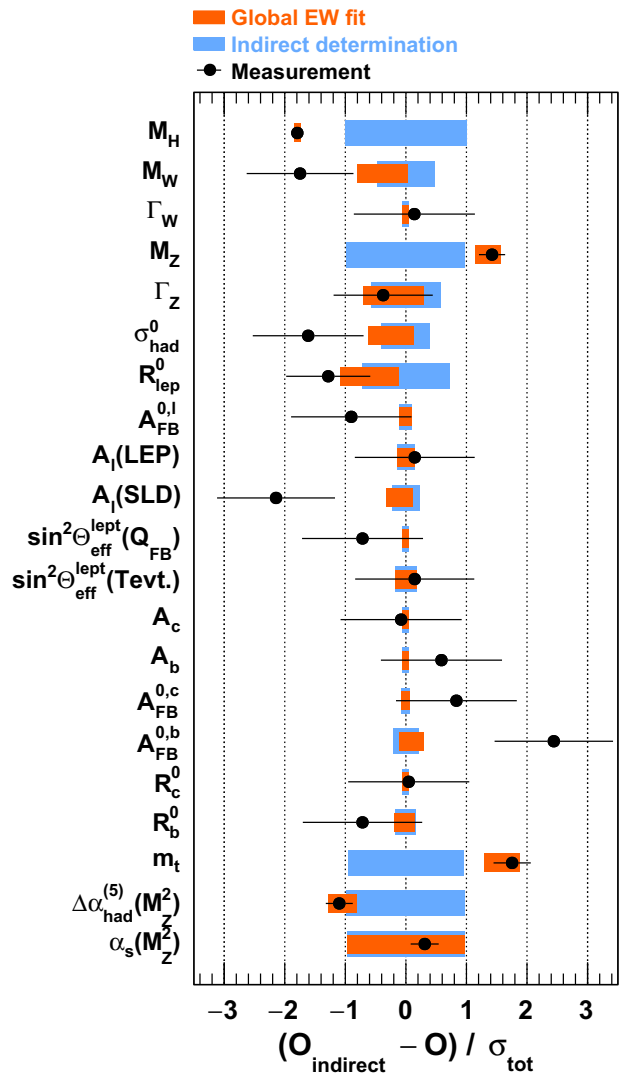

tions illustrate the impact of their uncertainties on the total uncertainties. The indirect determination of an observable corresponds to a fit without using the constraint from the corresponding input measurement

of the plot is included. The results are compared to the direct $M_{H}$ measurement as well as to the result of a fit including all data except the direct $M_{H}$ measurement. This latter fit gives the indirect determination

$M_{H}=90_{-18}^{+21} \mathrm{GeV}$,

which is in agreement with the direct measurement within 1.7 standard deviations. The value is lower by $3 \mathrm{GeV}$ than in our previous result $\left(93_{-21}^{+25} \mathrm{GeV}\right)$ [23] due to the lower value of $m_{t}$ used here. The reduced uncertainty of ${ }_{-18}^{+21} \mathrm{GeV}$ compared to ${ }_{-21}^{+25} \mathrm{GeV}$ previously, is due to the smaller uncertainty in $m_{t}$. When assuming perfect knowledge of $m_{t}, \Delta \alpha_{\mathrm{had}}^{(5)}\left(M_{Z}^{2}\right)$ and $\alpha_{S}\left(M_{Z}^{2}\right)$, the uncertainty is reduced by ${ }_{-3.5}^{+4.5},{ }_{-4}^{+5}$ and $\pm 2 \mathrm{GeV}$, respectively. The predictions of $M_{H}$ using $A_{\ell}, A_{\mathrm{FB}}^{0, b}$ and $M_{W}$ (LEP and Tevatron) concur with earlier findings [9]. The predictions derived from the ATLAS $M_{W}$ and Tevatron $\sin ^{2} \theta_{\text {eff }}^{\ell}$ measurements are in agreement with the direct $M_{H}$ measurement. 


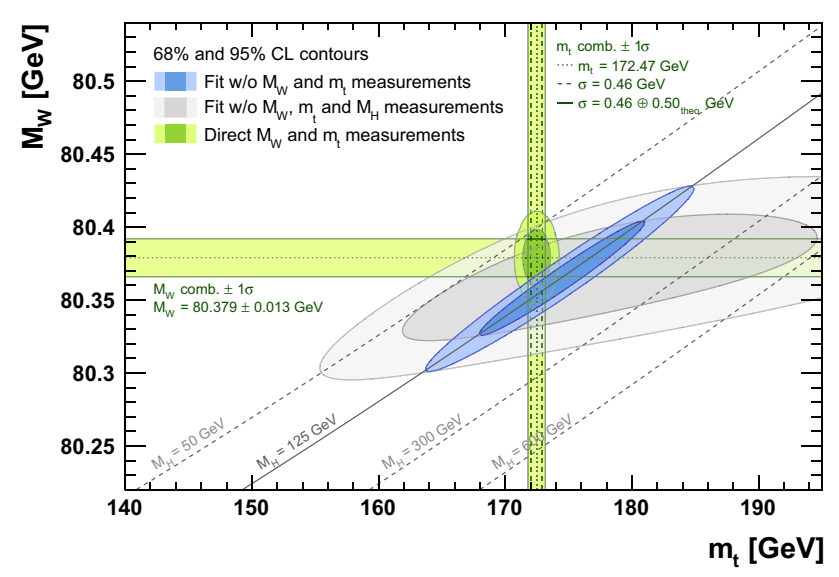

Fig. 5 Contours at 68 and 95\% CL obtained from scans of $M_{W}$ versus $m_{t}$ for the fit including (blue) and excluding the $M_{H}$ measurement (grey), as compared to the direct measurements (green vertical and horizontal $1 \sigma$ bands, and two-dimensional $1 \sigma$ and $2 \sigma$ ellipses). The direct measurements of $M_{W}$ and $m_{t}$ are excluded from the fits

An important consistency test of the SM is the simultaneous indirect determination of $m_{t}$ and $M_{W}$. A scan of the confidence level (CL) profile of $M_{W}$ versus $m_{t}$ is shown in Fig. 5 for the scenarios where the direct $M_{H}$ measurement is included in the fit (blue) or not (grey). Both contours agree with the direct measurements (green bands and ellipse for two degrees of freedom).

Figure 6 displays $\Delta \chi^{2}$ fit profiles for the indirect determination of some of the electroweak observables. ${ }^{4}$ The results are shown for fits including (blue) and excluding (grey) the direct $M_{H}$ measurement highlighting the strong impact of the $M_{H}$ measurement on the fit constraints. The direct measurement of each observable with its $1 \sigma$ uncertainty are indicated by the data points at $\Delta \chi^{2}=1$. The detailed predictions of the fit are given in Table 1 .

The fit indirectly determines the $W$ mass to be

$$
\begin{aligned}
M_{W}= & 80.3535 \pm 0.0027_{m_{t}} \pm 0.0030_{\delta_{\text {theo }} m_{t}} \\
& \pm 0.0026_{M_{Z}} \pm 0.0026_{\alpha_{S}} \\
& \pm 0.0024_{\Delta \alpha_{\text {had }}} \pm 0.0001_{M_{H}} \pm 0.0040_{\delta_{\text {theo }} M_{W}} \mathrm{GeV}, \\
= & 80.354 \pm 0.007_{\text {tot }} \mathrm{GeV}
\end{aligned}
$$

where the the different uncertainty contributions originate from the uncertainties on the input values of the fit. ${ }^{5}$ The effective leptonic weak mixing angle is determined as

\footnotetext{
4 The indirect determination profiles are obtained by excluding the input measurement of the respective observable from the fit (see figure legends).

${ }^{5}$ In our previous work [23] the uncertainty contributions from $\Delta \alpha_{\text {had }}^{(5)}\left(M_{Z}^{2}\right)$ and $\alpha_{S}\left(M_{Z}^{2}\right)$ have been underestimated due to numerical instabilities, introduced by an insufficent number of sampling points. The correct values are 0.0026 , which reduces to 0.0024 for $\Delta \alpha_{\text {had }}^{(5)}\left(M_{Z}^{2}\right)$ with the current input, and 0.0027 , which becomes 0.0026 for $\alpha_{S}\left(M_{Z}^{2}\right)$.
}

$$
\begin{aligned}
\sin ^{2} \theta_{\text {eff }}^{\ell}= & 0.231532 \pm 0.000011_{m_{t}} \pm 0.000016_{\delta_{\text {theo }} m_{t}} \\
& \pm 0.000012_{M_{Z}} \pm 0.000021_{\alpha_{S}} \\
& \pm 0.000035_{\Delta \alpha_{\text {had }}} \pm 0.000001_{M_{H}} \\
& \pm 0.000040_{\delta_{\text {theo }} \sin ^{2} \theta_{\text {eff }}^{\ell},}, \\
= & 0.23153 \pm 0.00006_{\text {tot }} .
\end{aligned}
$$

When evaluating $\sin ^{2} \theta_{\mathrm{eff}}^{\ell}$ through the parametric formula from Ref. [71], an upward shift of $2 \cdot 10^{-5}$ with respect to the fit result is observed, mostly due to the inclusion of $M_{W}$ in the fit. Using the parametric formula the total uncertainty is larger by $0.6 \cdot 10^{-5}$, as the global fit exploits the additional constraint from $M_{W}$. The fit also constrains the nuisance parameter associated with the theoretical uncertainty in the calculation of $\sin ^{2} \theta_{\text {eff }}^{\ell}$, resulting in a reduced theoretical uncertainty of $4.0 \cdot 10^{-5}$ compared to the $4.7 \cdot 10^{-5}$ input uncertainty.

While the indirect determinations are about a factor of two more precise than the corresponding measurements, new measurements of $M_{W}$ and $\sin ^{2} \theta_{\mathrm{eff}}^{\ell}$ at the LHC are expected to improve the experimental precision in the coming years. It is thus desirable to also improve the indirect determinations. The largest individual uncertainties are of theoretical nature due to missing higher order calculations and the interpretation of the kinematic $m_{t}$ measurements. The second largest source of uncertainty in the prediction of $\sin ^{2} \theta_{\mathrm{eff}}^{\ell}$ stems from $\Delta \alpha_{\text {had }}^{(5)}\left(M_{Z}^{2}\right)$, for which improvements would also lead to more precise predictions of $M_{W}$ and $M_{H}$. Once these uncertainties have been reduced, a more precise measurement of $m_{t}$ and external input on $\alpha_{S}\left(M_{Z}^{2}\right)$ will help to raise the precision further.

The mass of the top quark is indirectly determined to be

$m_{t}=176.4 \pm 2.1 \mathrm{GeV}$,

with a theoretical uncertainty of $0.6 \mathrm{GeV}$ induced by the theoretical uncertainty on the prediction of $M_{W}$. The largest potential to improve the precision of the indirect determination of $m_{t}$ is through a more precise measurement of $M_{W}$. Perfect knowledge of $M_{W}$ would result in an uncertainty on $m_{t}$ of $0.9 \mathrm{GeV}$.

The strong coupling strength at the $Z$-boson mass scale is determined to be

$\alpha_{S}\left(M_{Z}^{2}\right)=0.1194 \pm 0.0029$,

which corresponds to a determination at full next-to-next-to leading order (NNLO) for electroweak and strong contributions, and partial strong next-to-NNLO (NNNLO) corrections. The theory uncertainty of this result is 0.0009 , which is shared in equal parts between missing higher orders in the calculations of the radiator functions and the partial widths of the $Z$ boson. The most important constraints on $\alpha_{S}\left(M_{Z}^{2}\right)$ come from the measurements of $R_{\ell}^{0}, \Gamma_{Z}$ and $\sigma_{\text {had }}^{0}$, also shown in Fig. 6. The values of $\alpha_{S}\left(M_{Z}^{2}\right)$ obtained from the individual 

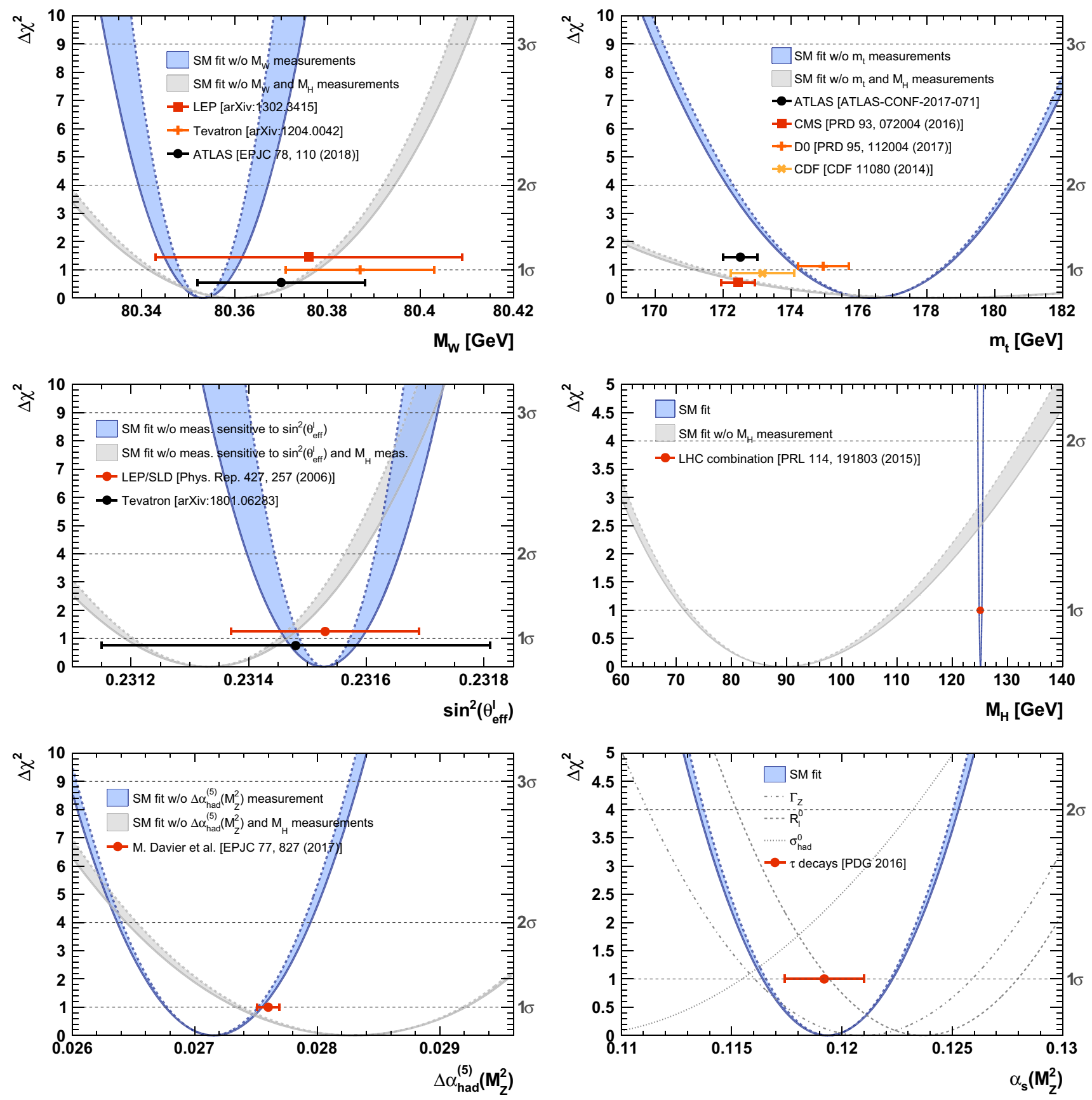

Fig. 6 Scans of $\Delta \chi^{2}$ as a function of $M_{W}$ (top left), $m_{t}$ (top right), $\sin ^{2} \theta_{\text {eff }}^{\ell}$ (middle left), $M_{H}$ (middle right), $\Delta \alpha_{\text {had }}^{(5)}\left(M_{Z}^{2}\right)$ (bottom left) and $\alpha_{S}\left(M_{Z}^{2}\right)$ (bottom right), under varying conditions. The results of the fits without and with the measurement of $M_{H}$ as input are shown in grey

measurements are $0.1237 \pm 0.0043\left(R_{\ell}^{0}\right), 0.1209 \pm 0.0049$ $\left(\Gamma_{Z}\right)$ and $0.1078 \pm 0.0076\left(\sigma_{\text {had }}^{0}\right)$. A fit to all three measurements results in a value of $0.1203 \pm 0.0030$, which is only slightly less precise than the result of the full fit. The results obtained for $\alpha_{S}\left(M_{Z}^{2}\right)$ are stable with respect to additional invisible beyond-the-standard-model contributions to $\Gamma_{Z}$.

and blue colours, respectively. The solid and dotted lines represent the results when including or excluding the theoretical uncertainties. The data points with uncertainty bars indicate the direct measurements of a given observable

No significant deviation from the direct measurements is observed in any of these predictions. The indirect determinations of $M_{W}$ and $\sin ^{2} \theta_{\text {eff }}^{\ell}$ outperform the direct measurements in precision while the indirect determinations of $m_{t}$ and $\alpha_{S}\left(M_{Z}^{2}\right)$ are competitive to other experimental results. 


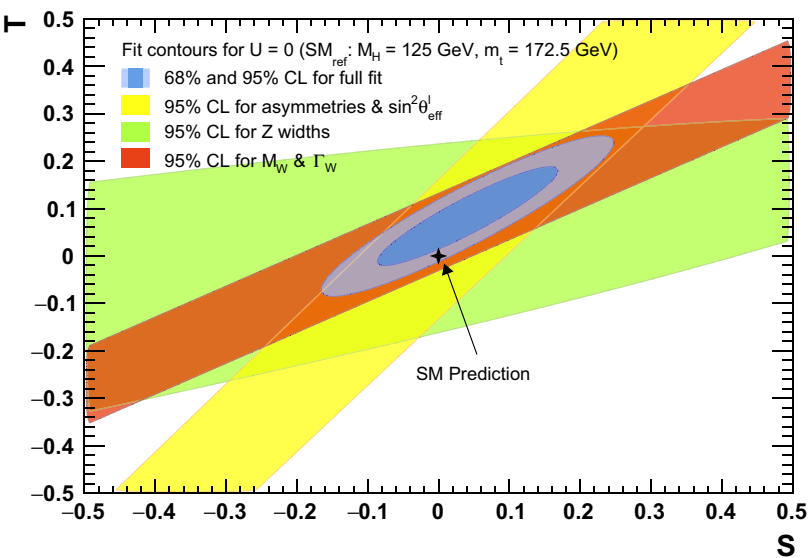

Fig. 7 Constraints in the oblique parameters $S$ and $T$, with the $U$ parameter fixed to zero, using all observables (blue). Individual constraints are shown from the asymmetry and direct $\sin ^{2} \theta_{\text {eff }}^{\ell}$ measurements (yellow), the $Z$ partial and total widths (green) and $W$ mass and width (red), with confidence levels drawn for one degree of freedom

\section{Oblique parameters}

Using the updated SM reference values $M_{H \text {,ref }}=125 \mathrm{GeV}$ and $m_{t \text {,ref }}=172.5 \mathrm{GeV}$ we obtain for the oblique parameters denoted $S, T, U[92,93]$ the following values:

$S=0.04 \pm 0.11, \quad T=0.09 \pm 0.14$,

$U=-0.02 \pm 0.11$,

with correlation coefficients of +0.92 between $S$ and $T$, $-0.68(-0.87)$ between $S$ and $U(T$ and $U)$. Fixing $U=0$ one obtains $\left.S\right|_{U=0}=0.04 \pm 0.08$ and $\left.T\right|_{U=0}=0.08 \pm 0.07$, with a correlation coefficient of +0.92 . The values are very close to the ones obtained in our previous work [23]. Small numerical differences result from a change of the SM reference point from $m_{t \text {, ref }}=173 \mathrm{GeV}$ to $172.5 \mathrm{GeV}$. The constraints on $S$ and $T$ for a fixed value of $U=0$ are shown in Fig. 7.

\section{Global fits in the two-Higgs-doublet model}

Combining information from the electroweak precision data, Higgs boson coupling measurements, flavour observables and the anomalous magnetic moment of the muon we derive in this section constraints on parameters of various $2 \mathrm{HDM}$ scenarios.

Besides the four mass parameters for the scalars, $M_{h}, M_{H}$, $M_{A}$, and $M_{H^{ \pm}}$, the 2HDM introduces the angle $\alpha$, which describes the mixing of the two neutral Higgs fields $h$ and $H$, and the angle $\beta$ that fixes the ratio of the vacuum expectation values of the two Higgs doublets, $\tan \beta=v_{2} / v_{1}$. We only consider $2 \mathrm{HDM}$ scenarios with $\mathrm{a} \mathbb{Z}_{2}$ symmetric potential with a dimension-two softly broken term proportional to the scale parameter $M_{12}^{2}$.

Depending on the Yukawa couplings of the two Higgs doublets, the 2HDM may introduce dangerous flavour-changing neutral currents (FCNCs) and CP violating interactions. CP conservation can be maintained by fixing the Higgs boson couplings for up-type quarks, down-type quarks, and leptons to specific values [27,94]. In this work, four CP conserving 2HDM scenarios are studied. In the Type-I scenario, only one of the two Higgs doublets is allowed to couple to fermions, while the other couples to the gauge bosons. The Type-II scenario is defined by a separation of the Yukawa interactions: one Higgs doublet couples only to up-type quarks and the other only to down-type quarks and charged leptons. The Type-II 2HDM resembles the Higgs sector in the Minimal Supersymmetric Standard Model. The third, lepton specific scenario is similar to the Type-I model with the difference that leptons only couple to the other Higgs doublet that does not interact with the quarks. Finally, the fourth, flipped scenario is the same as the Type-II model with swapped lepton couplings to the Higgs doublets.

Throughout this section the lightest scalar Higgs boson, $M_{h}$, is identified with the observed Higgs boson with mass fixed to $125.09 \pm 0.24 \mathrm{GeV}$ [18]. If not stated otherwise, all other 2HDM model parameters are allowed to vary within the intervals: $130<M_{H}, M_{A}<1000 \mathrm{GeV}, 100<M_{H^{ \pm}}<$ $1000 \mathrm{GeV}, 0 \leq \beta-\alpha \leq \pi, 0.001<\tan \beta<50$, and $-8 \cdot 10^{5}<M_{12}^{2}<8 \cdot 10^{5} \mathrm{GeV}^{2}$. No contribution from new physics other than the 2HDM is assumed.

Direct searches for additional Higgs bosons in collider experiments can be interpreted in the context of the 2HDM (see, for example, Refs. [95,96]). However, due to the large freedom in the choice of the 2HDM parameters, these search results provide only weak absolute exclusion limits on the masses of the scalars. From searches for a charged Higgs boson by the LEP experiments [28] a lower limit of $M_{H^{ \pm}}>$ $72.5 \mathrm{GeV}$ was reported for the Type-I scenario, while a limit of $M_{H^{ \pm}} \gtrsim 150 \mathrm{GeV}$ can be derived from searches at the LHC for the Type-II scenario [95]. Stronger mass limits mainly on $M_{H^{ \pm}}$can be obtained for specific regions of $\tan \beta$.

\subsection{Constraints from Higgs boson coupling measurements}

A second Higgs doublet modifies the coupling strengths of the lightest neutral Higgs boson $h$ to SM particles compared to those of the SM Higgs boson. The modifications depend on the 2HDM scenario and parameters in particular the angles $\alpha$ and $\beta$. Constraints on $h$ are derived from the joint ATLAS and CMS Higgs boson coupling analysis [97] in which measurements sensitive to five Higgs boson production modes (ggF, VBF, $W H, Z H, t \bar{t} H)$ and five decay modes $(\gamma \gamma, W W, Z Z, \tau \tau, b \bar{b})$ were combined. We make use of the relative signal strengths $\mu_{i j}$ defined as the ratio 

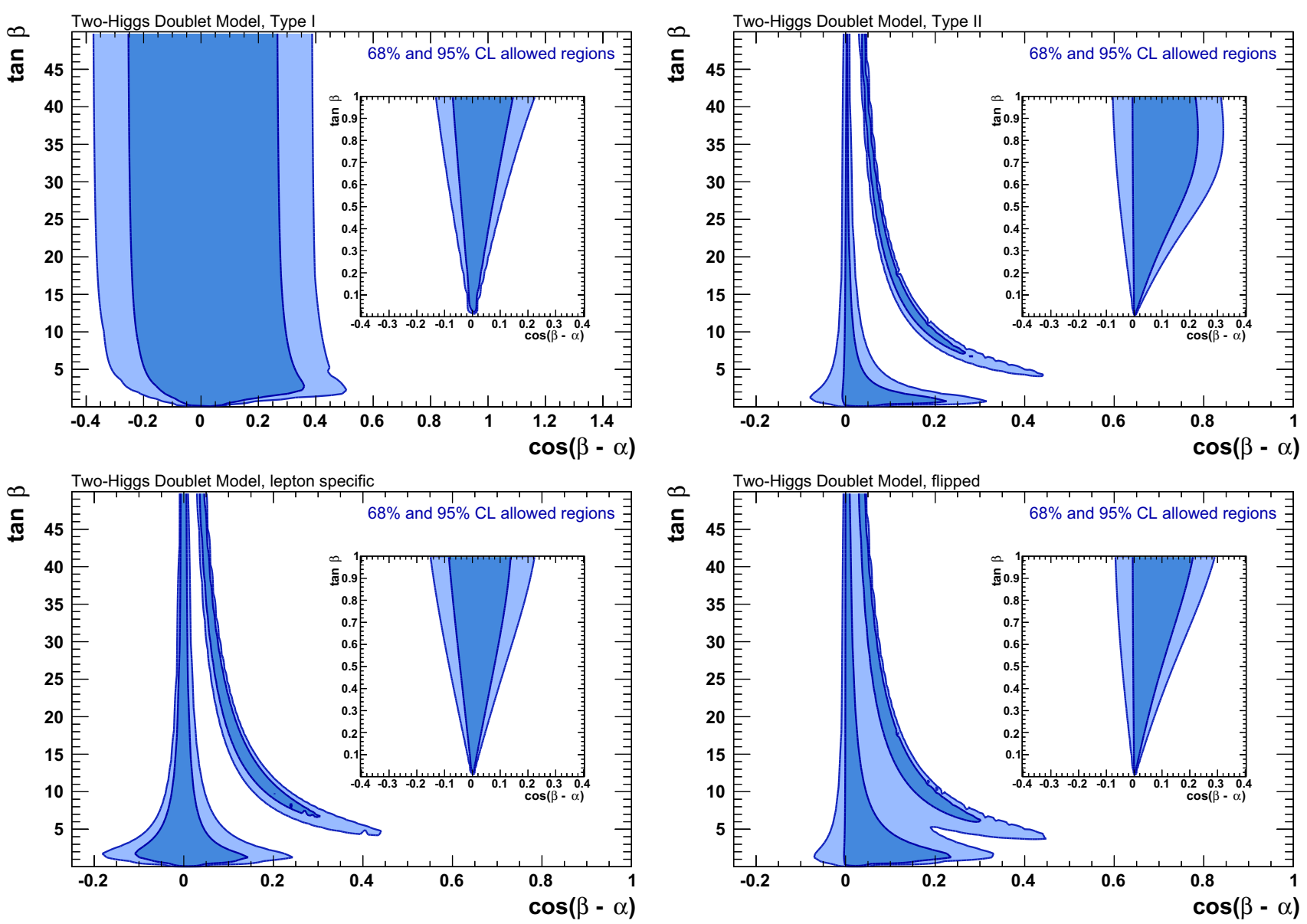

Fig. 8 Results from 2HDM fits using the ATLAS and CMS combined Higgs coupling strength measurements. Shown are allowed parameter regions ( 68 and $95 \% \mathrm{CL}$ ) for the four $2 \mathrm{HDM}$ scenarios from scans of

of measured over predicted cross section times branching ratio, $\mu_{i j}=\left(\sigma_{i} \cdot \mathcal{B}_{j}\right) /\left(\sigma_{i}^{\mathrm{SM}} \cdot \mathcal{B}_{j}^{\mathrm{SM}}\right)$. We include the 20 (out of the 25 possible) $\mu_{i j}$ parameters determined by ATLAS and CMS together with their uncertainties and correlations. A validation of our results is discussed in the Appendix on page 24 .

The corresponding SM predictions and uncertainties are taken from Ref. [98]. The signal strength measurements are compared with the theory predictions for the $2 \mathrm{HDM}$ calculated with the program $2 \mathrm{HDMC}$ [99]. ${ }^{6}$ In the calculation of the $\mu_{i j}$ for the $2 \mathrm{HDM}$ also the denominator $\sigma_{i}^{\mathrm{SM}} \cdot \mathcal{B}_{j}^{\mathrm{SM}}$ is determined using $2 \mathrm{HDMC}$ for consistency. Since more precise theory predictions for the SM cross sections and branching ratios exist and are used for the normalisation of the results in

\footnotetext{
6 2HDMC computes the couplings of all five Higgs boson states to SM particles for a given set of parameters in a CP conserving 2HDM with general Yukawa structure. From these couplings, production and decay rates of the Higgs boson states can be derived. Most decay widths are calculated at leading QCD order in 2HDMC. Higher order QCD corrections are included for couplings to fermion and gluon pairs.
}

$\tan \beta$ versus $\cos (\beta-\alpha)$ : Type-I (top left), Type-II (top right), lepton specific (bottom left) and flipped (bottom right) 2 HDMs. The figure insets show a zoom of the region with $\tan \beta<1$

[97], theory uncertainties in the SM prediction from [98] are taken into account as additional scaling (nuisance) parameters of the $\mu_{i j}$.

The constraints from the Higgs boson signal strength measurements on the four 2HDM scenarios are shown as 68 and 95\% CL allowed regions in the $\tan \beta$ versus $\cos (\beta-\alpha)$ plane in Fig. $8{ }^{7}$

The angles $\alpha$ and $\beta$ are highly constrained in all 2HDM scenarios except for Type-I. The allowed parameter regions are concentrated in two bands corresponding to solutions with $\beta \pm \alpha=\pi / 2$. For $\beta-\alpha=\pi / 2$, the Yukawa structure of the SM is reproduced (alignment limit). The case $\beta+\alpha=\pi / 2$ differs from the SM-like Yukawa couplings by a sign flip that is still allowed by the combined coupling strengths measurements. These constraints are differently

\footnotetext{
7 Theoretical bounds from positivity of the Higgs potential, tree-level unitarity, and perturbativity of the quartic Higgs boson couplings as implemented in $2 \mathrm{HDMC}$ were found to give no additional constraints in these figures.
} 
Table 2 Flavour physics observables and values used in the 2HDM fit

\begin{tabular}{lll}
\hline Observable & Values & References \\
\hline $\mathcal{B}\left(B \rightarrow X_{s} \gamma\right)$ for $E_{\gamma}>1.6 \mathrm{GeV}$ & $\left(3.32 \pm 0.15_{\text {stat }+ \text { syst }}\right) \cdot 10^{-4} \pm 7 \%_{\text {theo }}$ & {$[101-103]$} \\
$R(D)$ & $0.407 \pm 0.039_{\text {stat }} \pm 0.024_{\text {syst }} \pm 0.008_{\text {theo }}$ & {$[101,104]$} \\
$R\left(D^{*}\right)$ & $0.304 \pm 0.013_{\text {stat }} \pm 0.007_{\text {syst }} \pm 0.003_{\text {theo }}$ & {$[101,105]$} \\
$\mathcal{B}(B \rightarrow \tau \nu)$ & $(1.06 \pm 0.19) \cdot 10^{-4}$ & {$[101]$} \\
$\mathcal{B}\left(B_{s} \rightarrow \mu \mu\right)(\mathrm{CMS})$ & $\left(2.8_{-0.9}^{+1.0}\right) \cdot 10^{-9}$ & {$[106]$} \\
$\mathcal{B}\left(B_{s} \rightarrow \mu \mu\right)(\mathrm{LHCb})$ & $\left(3.0 \pm 0.6_{\text {stat }}^{+0.3}{ }_{-2 \mathrm{sys}}\right) \cdot 10^{-9}$ & {$[107]$} \\
$\mathcal{B}\left(B_{d} \rightarrow \mu \mu\right)(\mathrm{CMS})$ & $\left(4.4_{-1.9}^{+2.2}\right) \cdot 10^{-10}$ & {$[106]$} \\
$\mathcal{B}\left(B_{d} \rightarrow \mu \mu\right)(\mathrm{LHCb})$ & $\left(1.5_{-1.0_{\text {stat }}^{+0.1}}^{+1.2}\right) \cdot 10^{-10}$ & {$[107]$} \\
$\mathcal{B}\left(D_{s} \rightarrow \mu \nu\right)$ & $\left(5.54 \pm 0.20_{\text {stat }} \pm 0.13_{\text {syst }}\right) \cdot 10^{-3}$ & {$[101]$} \\
$\mathcal{B}\left(D_{s} \rightarrow \tau \nu\right)$ & $\left(5.51 \pm 0.18_{\text {stat }} \pm 0.16_{\text {syst }}\right) \cdot 10^{-2}$ & {$[101]$} \\
$\Delta m_{d}$ & $(0.5065 \pm 0.0019) \mathrm{ps}^{-1}$ & {$[101]$} \\
$\Delta m_{s}$ & $(17.757 \pm 0.021) \mathrm{ps}^{-1}$ & {$[101]$} \\
$\mathcal{B}(K \rightarrow \mu \nu) / \mathcal{B}(\pi \rightarrow \mu \nu)$ & $0.6357 \pm 0.0011$ & {$[46]$}
\end{tabular}

pronounced in the four 2HDM scenarios as they depend on the Yukawa coupling strengths. In the Type-I scenario (top left panel in Fig. 8) the Yukawa couplings of $h$ to all fermions are proportional to $\cos \alpha / \sin \beta$. The constraints are stronger in the other three scenarios as the Yukawa coupling for at least one fermion type is proportional to $-\sin \alpha / \cos \beta$. In the flipped scenario (bottom right panel) only the Yukawa coupling to down-type quarks is given by $-\sin \alpha / \cos \beta$, which is constrained by the measurements of $H \rightarrow b \bar{b}$. Measurements of $H \rightarrow \tau^{+} \tau^{-}$give stronger bounds in the Type-II (top right panel) and lepton specific (bottom left panel) scenarios where the Yukawa coupling to leptons is given by $-\sin \alpha / \cos \beta$. In all scenarios, the measurements of Higgs boson decays to $W$ and $Z$ boson pairs disfavour large values of $\cos (\beta-\alpha)$. Similar constraints have been obtained by the ATLAS collaboration [100].

\subsection{Constraints from flavour observables}

Because tree-level FCNC transitions are forbidden by construction in the four 2HDM scenarios considered, flavour violation only arises at loop level by the exchange of a charged Higgs boson with observable strength depending on the parameters $M_{H^{ \pm}}$and $\tan \beta$.

\section{Experimental input data and theory calculation}

The flavour physics observables taken into account in our analysis are listed in Table 2 and briefly described below.

For the branching fraction of the radiative decay $\mathcal{B}(B \rightarrow$ $\left.X_{s} \gamma\right)$ with $E_{\gamma}>1.6 \mathrm{GeV}$ we use the value of the Heavy Flavour Averaging Group (HFLAV) [101] which combines measurements from the BABAR [108-110], Belle [111-
113], and CLEO [114] experiments. The prediction for $\mathcal{B}\left(B \rightarrow X_{s} \gamma\right)$ has been adopted from Ref. [103] and includes QCD corrections up to NNLO [115]. We make use of the code implementation kindly provided by Misiak.

HFLAV also combined measurements of the semileptonic decay ratios of neutral $B$ mesons $R\left(D^{(*)}\right)=\mathcal{B}\left(\bar{B}^{0} \rightarrow\right.$ $\left.D^{(*)+} \tau^{-} \bar{v}\right) / \mathcal{B}\left(\bar{B}^{0} \rightarrow D^{(*)+} \ell^{-} \bar{v}\right)$ by BABAR [116,117], Belle [118-120], and $\mathrm{LHCb}$ [121] with a correlation of -0.23 between the two observables that is taken into account in the fit. The prediction of $R\left(D^{(*)}\right)[104,105,122]$ includes treelevel contributions of a charged Higgs boson and is based on form factors evaluated in Heavy-Quark Effective Theory. Variations of the parameters $\rho_{R(D)}^{2}, \rho_{R\left(D^{*}\right)}^{2}, R_{1}(1)$, and $R_{2}(1)$ are included in the fit with values and correlations taken from Ref. [101].

For the branching ratio $\mathcal{B}(B \rightarrow \tau \nu)$ we use the HFLAV average [101] of measurements from BABAR [123] and Belle [124]. For the prediction of $\mathcal{B}(B \rightarrow \tau \nu)$ in the 2HDM we use the calculation from Ref. [125], which contains treelevel contributions of a charged Higgs boson where the leading $\tan \beta$ corrections are resummed to all orders [125]. The theoretical uncertainties in $\left|V_{u b}\right|$ and $f_{B_{d}}$ (see below) are included.

The latest measurements of $\mathcal{B}\left(B_{s} \rightarrow \mu \mu\right)$ and $\mathcal{B}\left(B_{d} \rightarrow\right.$ $\mu \mu$ ) from $\mathrm{LHCb}$ [107] are combined in our fits with the CMS result [106], assuming them uncorrelated. Their theoretical predictions in the $2 \mathrm{HDM}$ include NLO corrections given in Refs. [126,127]. The SM contribution to these observables are known up to three-loop level in QCD and include NLO electroweak corrections [128-130]. The predictions depend on the CKM matrix elements $\left|V_{t b}\right|$ and $\left|V_{t s}\right|$ or $\left|V_{t d}\right|$, respectively, and on the respective hadronic parameters $f_{B_{S}}$ and $f_{B_{d}}$. Uncertainties in these parameters are taken into account in the fit. 
Table 3 Parameters used in the fit to the flavour observables. Most values are taken from latest available version of the CKM fit [141]. For the CKM matrix element $\left|V_{u b}\right|$ we use the average of inclusive and exclusive measurements [143], while all other CKM matrix elements are calculated from the Wolfenstein parameters. The parameters related to the $R\left(D^{(*)}\right)$ measurements, $\rho_{R(D)}^{2}, \rho_{R\left(D^{*}\right)}^{2}, R_{1}(1), R_{2}(1)$ are taken from Ref. [101]. Value and uncertainty for $\delta_{\mathrm{EM}}^{K / \pi}$ are taken from Ref. [144]

\begin{tabular}{llll}
\hline Parameter & Values & Parameter & Values \\
\hline$A$ & $0.8250_{-0.0111}^{+0.0071}$ & $f_{D_{s}}$ & $\left(248.2 \pm 0.3_{\text {stat }} \pm 1.9_{\text {syst }}\right) \mathrm{MeV}$ \\
$\lambda$ & $0.22509_{-0.00028}^{+0.00029}$ & $f_{B_{s}}$ & $\left(225.6 \pm 1.1_{\text {stat }} \pm 5.4_{\text {syst }}\right) \mathrm{MeV}$ \\
$\bar{\rho}$ & $0.1598_{-0.0072}^{+0.0076}$ & $f_{B_{s}} / f_{B_{d}}$ & $1.205 \pm 0.004_{\text {stat }} \pm 0.007_{\text {syst }}$ \\
$\bar{\eta}$ & $0.3499_{-0.0061}^{+0.0063}$ & $\hat{B}_{s}$ & $1.320 \pm 0.017_{\text {stat }} \pm 0.030_{\text {syst }}$ \\
$\left|V_{u b}\right|$ & $0.00395 \pm 0.00038_{\text {exp }} \pm 0.00039_{\text {theo }}$ & $\hat{B}_{s} / \hat{B}_{d}$ & $1.023 \pm 0.013_{\text {stat }} \pm 0.014_{\text {syst }}$ \\
$\rho_{R(D)}^{2}$ & $1.128 \pm 0.033$ & $\eta_{B}$ & $0.551 \pm 0.0022$ \\
$\rho_{R\left(D^{*}\right)}^{2}$ & $1.21 \pm 0.027$ & $f_{K} / f_{\pi}$ & $1.1952 \pm 0.0007_{\text {stat }} \pm 0.0029_{\text {syst }}$ \\
$R_{1}(1)$ & $1.404 \pm 0.032$ & $\delta_{\mathrm{EM}}^{K / \pi}$ & $-0.0070 \pm 0.0018$ \\
$R_{2}(1)$ & $0.854 \pm 0.020$ & & \\
\hline
\end{tabular}

The charged Higgs boson of the 2HDM contributes to the leptonic decays of $D_{s}$ mesons. For the observables $\mathcal{B}\left(D_{s} \rightarrow\right.$ $\mu \nu)$ and $\mathcal{B}\left(D_{s} \rightarrow \tau \nu\right)$ we use the HFLAV averages [101] of measurements from BABAR [131], Belle [132], and CLEO [133-135]. For the 2HDM predictions we use the analytic expression for the $2 \mathrm{HDM}$ tree-level contribution to $\mathcal{B}\left(D_{s} \rightarrow\right.$ $\ell v)$ from Ref. [136] that allows us to vary the dependencies on $\left|V_{c s}\right|$ and $f_{D_{s}}$ in the fit.

The charged Higgs boson also contributes via box diagrams to the mixing of the neutral $B_{d}$ and $B_{s}$ mesons altering the mixing frequencies $\Delta m_{d}$ and/or $\Delta m_{s}$. We use again the HFLAV [101] experimental averages for these quantities. Their predictions in the 2 HDM are obtained from analytic expressions of the full one-loop calculation of Refs. $[122,137]$ neglecting small terms proportional to $m_{b}^{2} / M_{W}^{2}$. The predictions depend on the CKM matrix elements $\left|V_{t d}\right|$ and $\left|V_{t s}\right|$, the bag parameters $\hat{B}_{d}$ and $\hat{B}_{s}$, and the decay constants $f_{B_{d}}$ and $f_{B_{s}}$, respectively, and the correction factor $\eta_{B}$.

Finally, the $2 \mathrm{HDM}$ contributes at leading order to the ratio $\mathcal{B}(K \rightarrow \mu \nu) / \mathcal{B}(\pi \rightarrow \mu \nu)$ for which we use a value adopted from Ref. [46], based on the measurement of the kaon decay rates [138], and the 2HDM prediction from Ref. [122]. The ratio involves the CKM matrix elements $\left|V_{u s}\right|$ and $\left|V_{u d}\right|$, the decay constants $f_{K}$ and $f_{\pi}$, and an electromagnetic correction $\delta_{\mathrm{EM}}^{K / \pi}$.

As input values for the unitarity CKM matrix we use the latest available results for the all-orders Wolfenstein parameters $A, \lambda, \bar{\rho}, \bar{\eta}$ from Refs. [139-141], taking them uncorrelated. A fully consistent analysis would require a combined fit of the Wolfenstein and 2HDM parameters within the 2HDM [142], which is however beyond the scope of this paper. Studies in Ref. [122] and by ourselves have shown that the numerical impact of the 2HDM on the CKM parameters is modest. For the CKM element
$\left|V_{u b}\right|$, occurring mainly in the prediction of the leptonic $B^{ \pm}$branching fraction, we take the average of inclusive and exclusive measurements [143] instead of the CKM fit prediction to allow for a more conservative uncertainty in view of the tension between the inclusive and exclusive results.

The input parameters used in the fit are summarised in Table 3 .

\section{Results}

Since most flavour observables are only sensitive to $M_{H^{ \pm}}$and $\tan \beta$, separate scans of these parameters are performed for each observable. The other 2HDM parameters are ignored in these scans, with the exception of $\mathcal{B}\left(B_{S / d} \rightarrow \mu \mu\right)$, where in addition $M_{H}, M_{A}$, and $M_{12}^{2}$ are allowed to float freely within the bounds defined in the introduction of Sect. 3 as these two observables depend at NLO level on these parameters. In all fits the CKM matrix elements and the other parameters given in Table 3 are allowed to vary within their uncertainties.

Figure 9 shows for the four 2HDM scenarios the onesided $95 \% \mathrm{CL}$ excluded regions in the $\tan \beta$ versus $M_{H^{ \pm}}$ plane as obtained from fits using the most sensitive individual flavour observables. The CLs are derived assuming a Gaussian behaviour of the test statistic with one degree of freedom. The Type-I (top left) and lepton specific (bottom left) scenarios are only weakly constrained allowing to exclude $\tan \beta<1$. Stronger constraints are obtained for the Type-II (top right) and flipped (bottom right) scenarios in which in particular $\mathcal{B}\left(B \rightarrow X_{s} \gamma\right)$ allows to exclude $M_{H^{ \pm}}<590 \mathrm{GeV}{ }^{8}$ Strong constraints

\footnotetext{
8 Our results are compatible with those of Ref. [145], where limits on $M_{H^{ \pm}}$between 570 and $800 \mathrm{GeV}$ are reported for the Type-II model,
} 

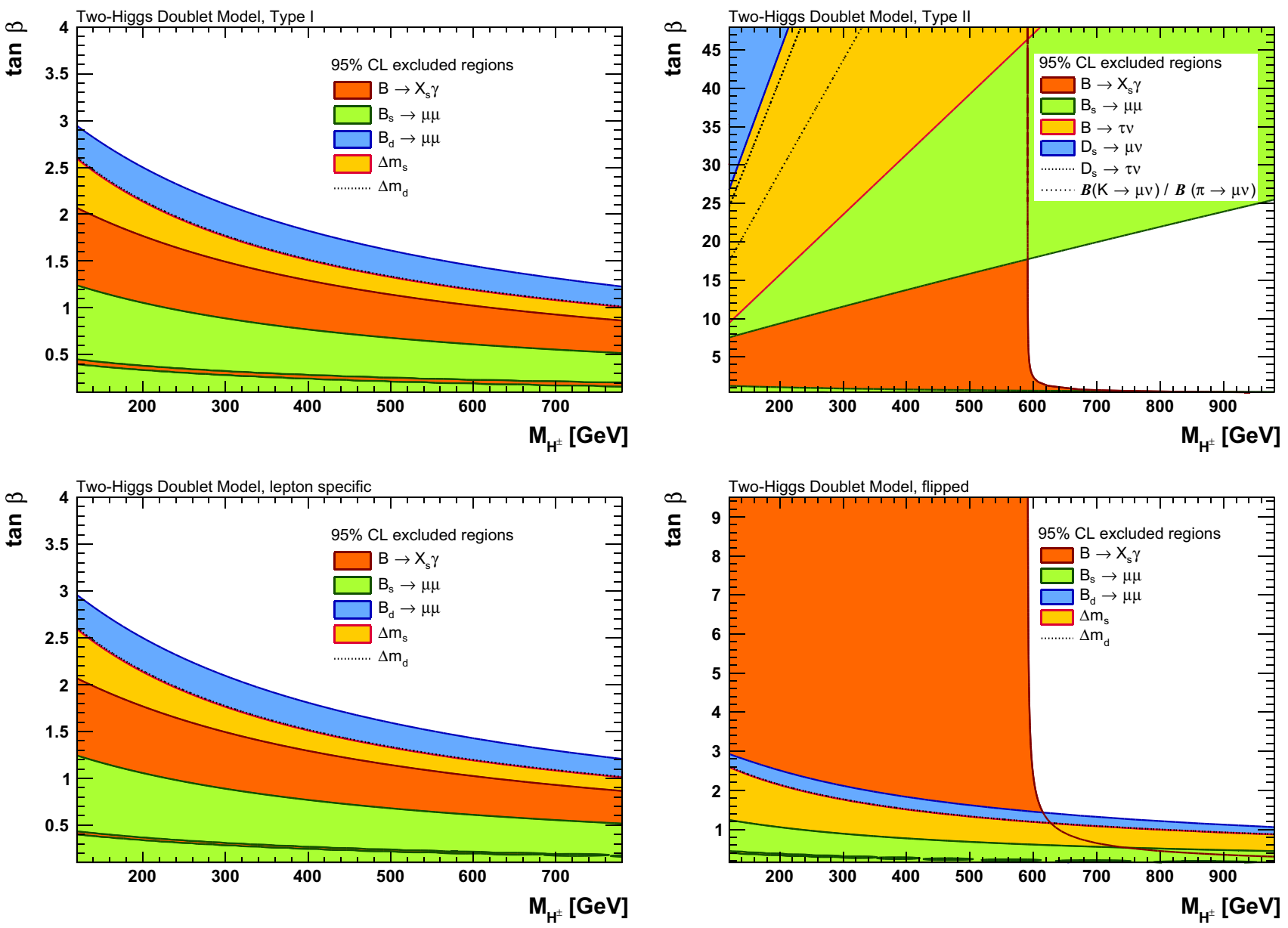

Fig. 9 Excluded parameter regions $(95 \% \mathrm{CL})$ in the $\tan \beta$ versus $M_{H^{ \pm}}$plane from individual observables for the four 2HDM scenarios considered: Type-I (top left), Type-II (top right), lepton specific (bottom left), flipped (bottom right)

on the Type-II model are also obtained from $\mathcal{B}\left(B_{s} \rightarrow\right.$ $\mu \mu) \cdot{ }^{9}$

The measurements of $R(D)$ and $R\left(D^{*}\right)$ differ from their SM predictions $[104,105,148]$. In the $2 \mathrm{HDM}$ only the TypeII scenario features a compatible parameter region (at large $\tan \beta$ and relatively small $M_{H^{ \pm}}$, not shown in the upper right plot of Fig. 9), which is, however, excluded by several other observables. Similar results have been reported in Ref. [122]. Because of this incompatibility $R(D)$ and $R\left(D^{(*)}\right)$ are excluded from our analysis in the following.

\section{Footnote 8 continued}

depending on the statistical method used (the CL has a relatively weak gradient versus $M_{H^{ \pm}}$and thus exhibits a strong numerical sensitivity to the details of the interpretation). Similar exclusion limits on $M_{H^{ \pm}}$can be achieved in a complex 2HDM (C2HDM), which features additional mixing between the neutral CP-even and CP-odd Higgs bosons [146].

9 Note that Refs. [122,147] obtain weaker limits because of the use of a two-sided test statistic while we use a one-sided one. In addition, updated values of input parameters improve the limits, especially the new $\mathcal{B}\left(B_{s} \rightarrow \mu \mu\right)$ measurements and better known CKM matrix elements.
3.3 Constraints from the anomalous magnetic moment of the muon

The measured value of the anomalous magnetic moment of the muon $a_{\mu}=\left(g_{\mu}-2\right) / 2$ shows a long-standing tension with the SM prediction of $\Delta a_{\mu}=(268 \pm 63 \pm 43) \cdot 10^{-11}$ $[26,149]$, where the first uncertainty is due the the measurement and the second the prediction (see also the recent reanalysis in Ref. [150]). Loops involving 2HDM bosons can modify the coupling between photons and muons. We have adopted the two-loop 2HDM prediction of $\Delta a_{\mu}$ from Refs. [151,152], which depends on all 2HDM parameters. We make use of the code implementation kindly provided by Stöckinger-Kim.

Figure 10 shows the 68 and $95 \%$ CL allowed regions in the $\tan \beta$ versus $M_{H^{ \pm}}$plane for the four $2 \mathrm{HDM}$ scenarios using only $\Delta a_{\mu}$ as input. All other parameters of the 2HDM are left free to vary within their respective bounds. Compatibility is found in a narrow band with $\tan \beta \ll 1$ and $M_{H^{ \pm}}$below about $600 \mathrm{GeV}$ (depending on the scenario), as well as for a region with larger $\tan \beta$ that broadens with decreasing $M_{H^{ \pm}}$. 

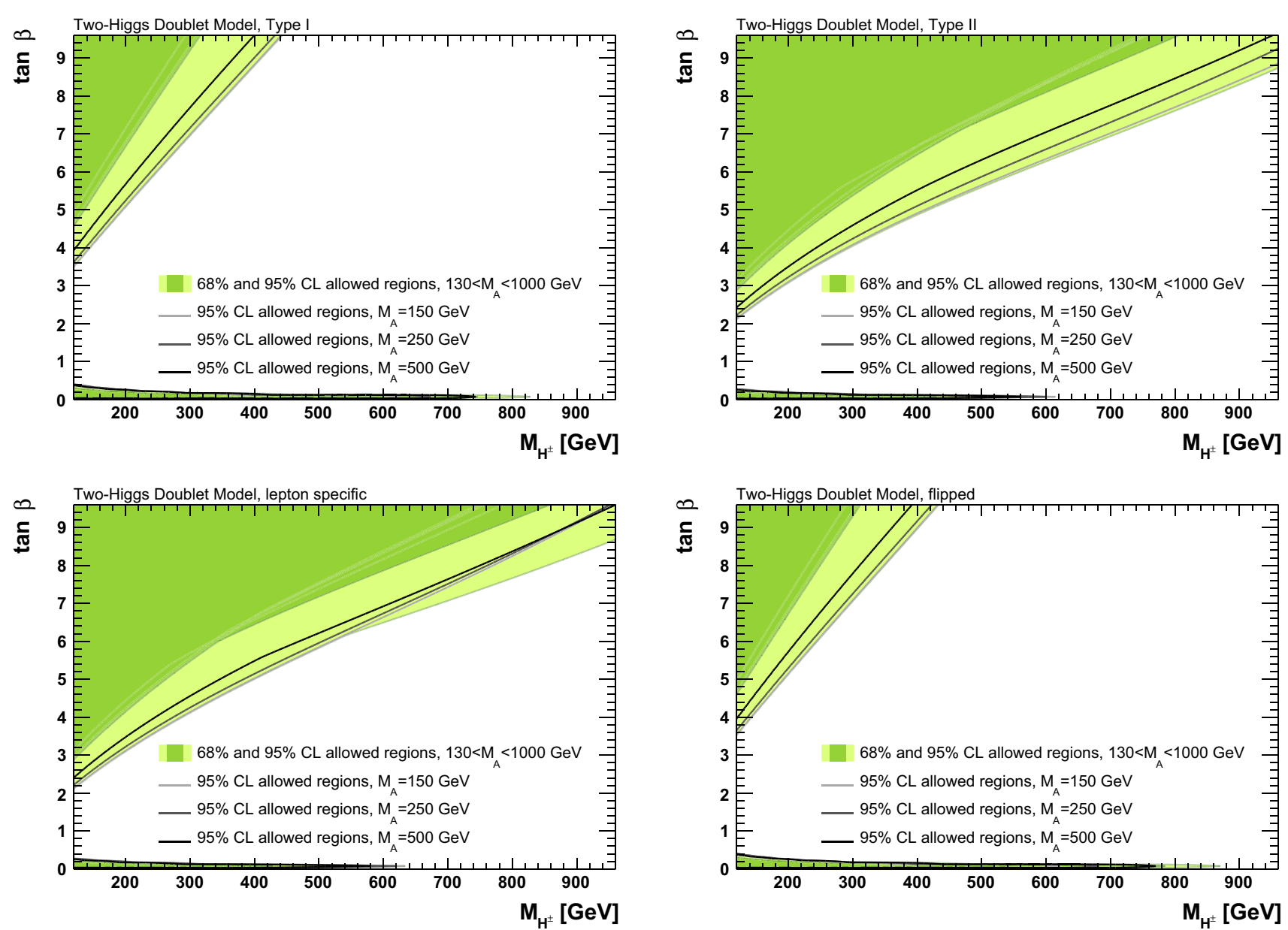

Fig. 10 2HDM fits using the anomalous magnetic moment of the muon as input. The green areas show allowed 68 and $95 \%$ CL regions in the $\tan \beta$ versus $M_{H^{ \pm}}$plane for freely floating $M_{A}$ and the four $2 \mathrm{HDM}$ sce-

narios considered: Type-I (top left), Type-II (top right), lepton specific (bottom left), and flipped (bottom right). The solid lines indicate $95 \%$ CL limits for different fixed values of $M_{A}$

The limits obtained from the $\Delta a_{\mu}$ measurement also depend on $M_{A}$. The allowed regions shown in Fig. 10 are mostly driven by the lower bound of $130 \mathrm{GeV}$ on $M_{A}$ in the scan, while for increasing values of $M_{A}$, the allowed regions in the $\tan \beta$ versus $M_{H^{ \pm}}$plane shrink. When combined with the constraints from the other flavour observables (cf. Fig. 9), values of $\tan \beta$ above $5 \sim 10$ remain allowed.

\subsection{Constraints from electroweak precision data}

The electroweak precision data can be used to constrain the $2 \mathrm{HDM}$ via the oblique parameters determined in Eq. (6). We use the predictions from Refs. [153-155] similar to our previous analysis [45]. The oblique corrections to electroweak observables in the 2HDM are independent of the Yukawa interactions and their impact is identical in the four 2HDM scenarios considered.

Figure 11 shows the 68 and 95\% CL allowed parameter regions in the neutral Higgs-boson mass plane $M_{A}$ versus

$M_{H}$ for fixed charged Higgs-boson masses of 250, 500, and $750 \mathrm{GeV}$ as obtained from fits using only the oblique parameters as input. All other parameters of the 2HDM (including $\beta-\alpha$ ) are free to vary in these scans. While no information on the absolute mass scale of the 2HDM bosons is obtained from the electroweak data, relative masses are constrained. In our previous analysis [45] we showed that the oblique parameters constrain the values of $M_{H}$ and $M_{A}$ to be close to $M_{H^{ \pm}}$for fixed $\beta-\alpha=\pi / 2$. Removing this restriction (cf. Fig. 11) relaxes the constraint to having either $M_{A}$ close to $M_{H^{ \pm}}$, or $M_{H}$ larger than $M_{H^{ \pm}}$.

\subsection{Combined fit}

We combine in this section the $2 \mathrm{HDM}$ constraints from the Higgs-boson coupling strength measurements, flavour observables, muon anomalous magnetic moment, and electroweak precision data. 


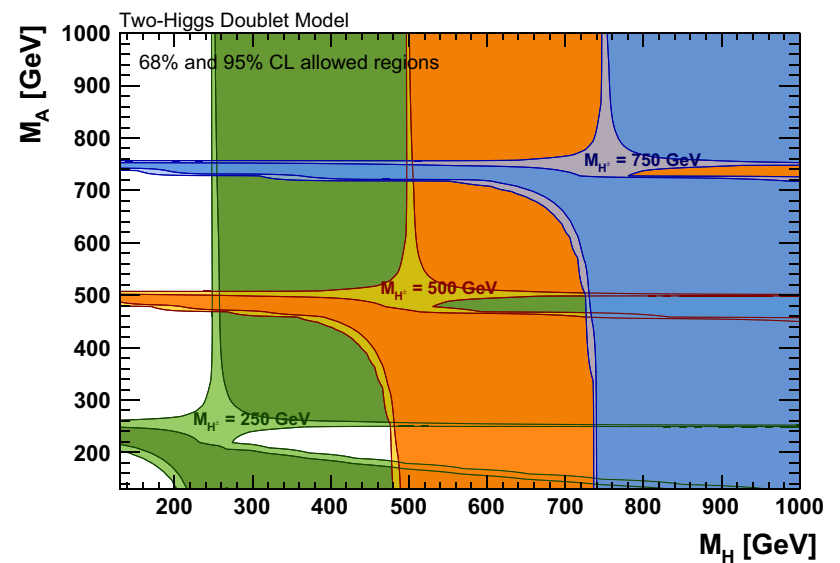

Fig. $112 \mathrm{HDM}$ fit results using the oblique $S, T, U$ parameters. Shown are allowed 68 and $95 \% \mathrm{CL}$ regions in the $M_{A}$ versus $M_{H}$ plane for fixed benchmark values of $M_{H^{ \pm}}$. The constraints are independent of the 2HDM scenario

Figure 12 shows for the four 2HDM scenarios considered the resulting 68 and $95 \% \mathrm{CL}$ allowed regions in the $M_{A}$ ver-
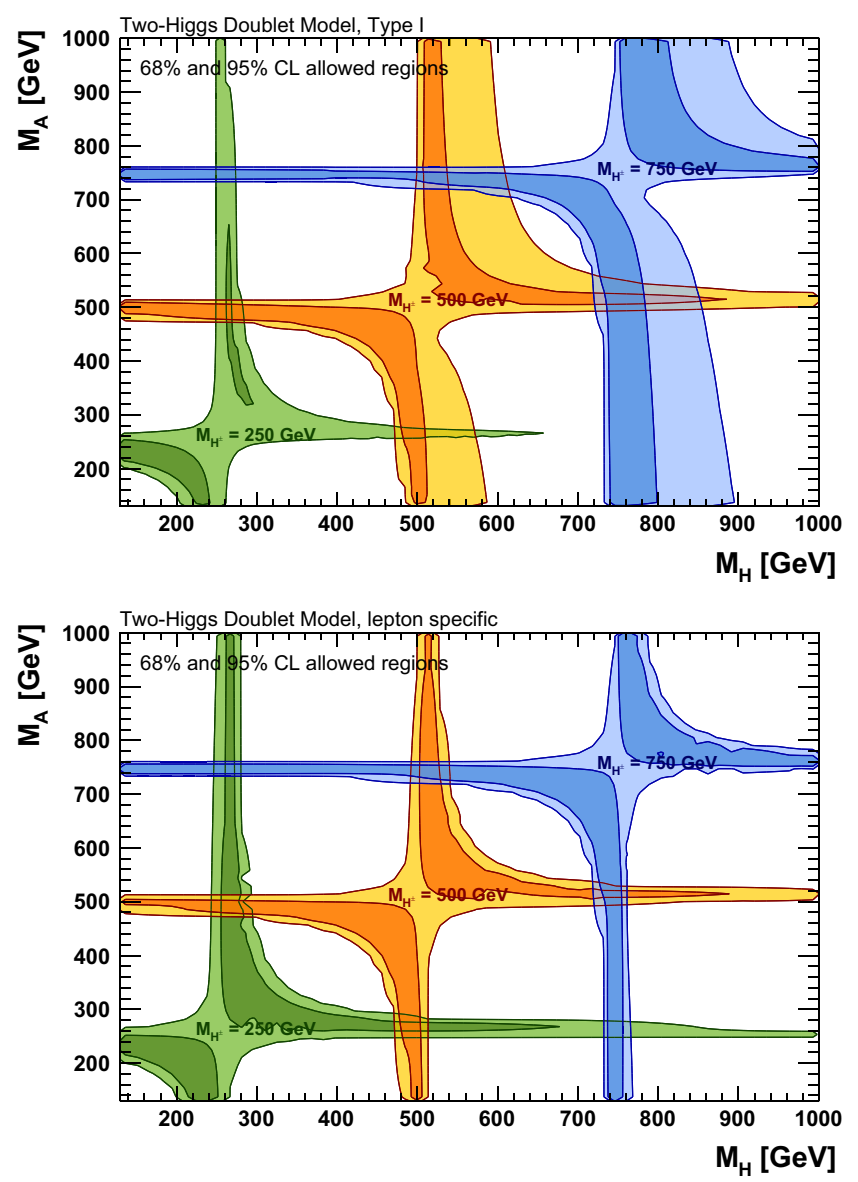

Fig. 12 2HDM fit results using a combination of constraints from the Higgs-boson coupling strength measurements, flavour observables, muon anomalous magnetic moment, and electroweak precision data. Shown are allowed 68 and 95\% CL regions in the $M_{A}$ versus $M_{H}$ plane sus $M_{H}$ plane for fixed (benchmark) charged Higgs-boson masses of 250,500 , and $750 \mathrm{GeV}$. All other 2HDM parameters are allowed to vary freely within their bounds. Depending on the 2HDM scenario and $M_{H^{ \pm}}$, the minimum $\chi^{2}$ values found lie between 48 and 59 for $N_{\text {dof }}=53$ (corresponding to $p$ values between 25 and $68 \%$ ).

The combined fit leads in all four 2HDM scenarios to a strong alignment of either the $H$ or the $A$ boson mass with that of the $H^{ \pm}$boson, owing to the constraint on $\beta-\alpha$ from the Higgs coupling strength measurements (cf. Fig. 8) in addition to those from the electroweak precision data. In this sense, the fit resembles the result from our previous analysis [45], but replacing the fixed restriction of $\beta-\alpha=\pi / 2$ by the Higgs couplings strengths measurements.

The absolute mass limits on $M_{\mathrm{H}^{ \pm}}$obtained from the flavour observables in the Type-II and flipped scenarios (cf. Fig. 9) exclude the low- $M_{\mathrm{H}^{ \pm}}$benchmarks, as indicated by the hatched regions in the two right-hand panels of Fig. 12 (where in addition different statistical assumptions are com-
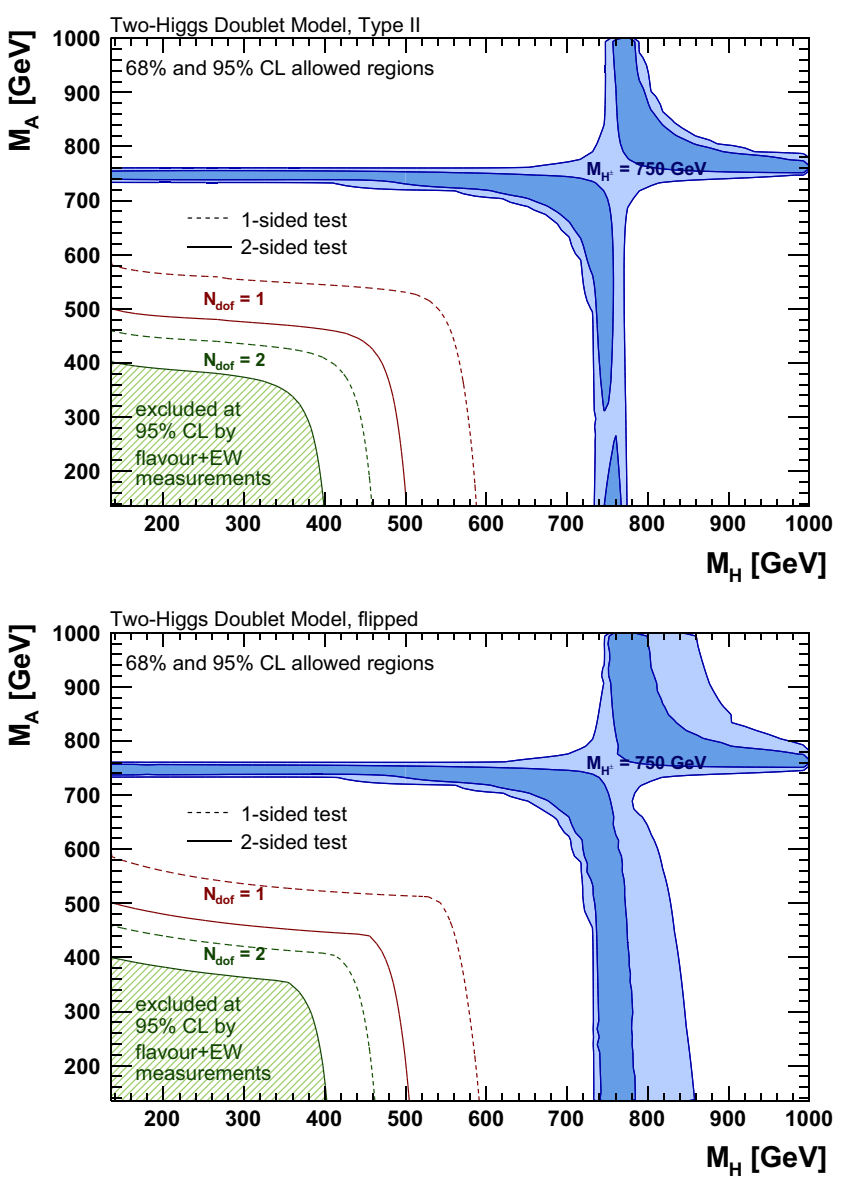

for fixed benchmark values of $M_{H^{ \pm}}$and for the four 2HDM scenarios considered: Type-I (top left), Type-II (top right), lepton specific (bottom left), and flipped (bottom right) 
pared: one-sided versus two-sided test statistic and one versus two degrees of freedom ${ }^{10}$ ). For these two scenarios pairs of $(H, A)$ masses below $\sim 400 \mathrm{GeV}$ are excluded for any set of values of the other 2HDM parameters. For the Type-I and lepton specific scenarios no absolute limits on the Higgs boson masses can be derived.

\section{Conclusion}

We have presented results for an updated global fit of the electroweak sector of the Standard Model using latest experimental and theoretical input. We include new precise kinematic top quark and $W$ boson mass measurements from the LHC, a $\sin ^{2} \theta_{\text {eff }}^{\ell}$ measurement from the Tevatron, and a new evaluation of the hadronic contribution to $\alpha\left(M_{Z}^{2}\right)$. The fit confirms the consistency of the Standard Model and slightly improves the precision of the indirect determination of key observables.

Using constraints from Higgs-boson coupling strength measurements, flavour observables, the muon anomalous magnetic moment, and electroweak precision data, we studied allowed and excluded parameter regions of four CP conserving two-Higgs-doublet models. Strong constraints on the extended Higgs boson masses are found for the so-called Type-II and flipped scenarios.

Acknowledgements We are indebted to Mikolaj Misiak and Hyejung Stöckinger-Kim for providing the implementation of their calculations of $\mathcal{B}\left(B \rightarrow X_{s} \gamma\right)$ and the muon anomalous magnetic moment in the two-Higgs-doublet models. We thank Rui Santos for helpful discussions and feedback on early stages of the paper. This work is supported by the German Research Foundation (DFG) in the Collaborative Research Centre (SFB) 676 "Particles, Strings and the Early Universe" located in Hamburg.

Open Access This article is distributed under the terms of the Creative Commons Attribution 4.0 International License (http://creativecomm ons.org/licenses/by/4.0/), which permits unrestricted use, distribution, and reproduction in any medium, provided you give appropriate credit to the original author(s) and the source, provide a link to the Creative Commons license, and indicate if changes were made.

Funded by SCOAP ${ }^{3}$.

\section{Appendix}

To validate our implementation of the Higgs boson coupling measurements with respect to the full result from the ATLAS and CMS combination [97], we have performed a fit of a generic new physics parametrisation. Here, new physics effects are assumed to uniformly vary the coupling strength of

\footnotetext{
10 The limits obtained for a two-sided test statistic and two degrees of freedom have been verified with a pseudo Monte Carlo study based on randomly drawn sets of the measurements used in the fit.
}

the Higgs boson to vector bosons and fermions, respectively, according to linear modifiers $\kappa_{V}$ and $\kappa_{F}$. No new particles are assumed to contribute to the Higgs boson production via loop diagrams and the branching fraction of the Higgs boson to unknown states is assumed to be zero. The constraints on $\kappa_{V}$ and $\kappa_{F}$ from the individual Higgs boson decay channels and their combination are shown in Fig. 13. We obtain the best fit values $\kappa_{V}=1.00 \pm 0.05$ and $\kappa_{F}=0.92 \pm 0.11$ with a correlation coefficient of -0.37 . Decent agreement with Ref. [97] is seen.

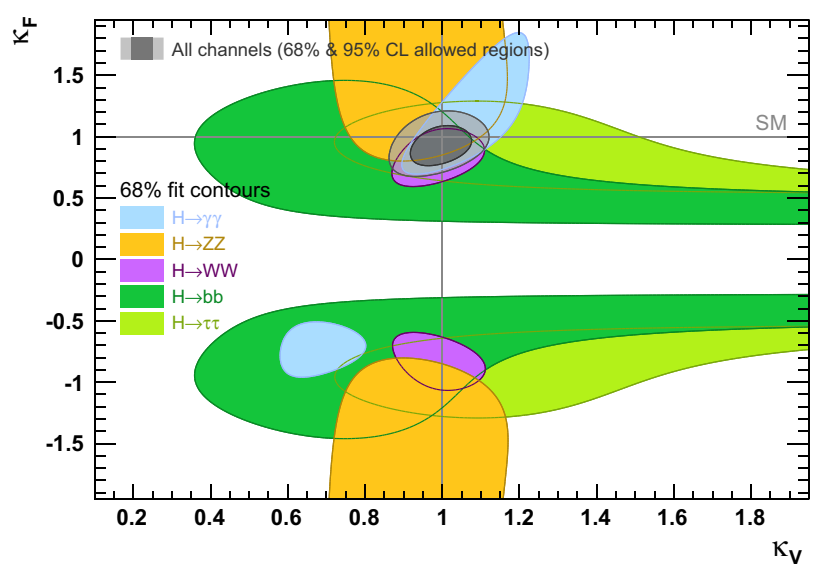

Fig. 13 Validation of our implementation of the combined ATLAS and CMS Higgs boson coupling measurements: preferred regions from a two-dimensional scan of the coupling strength modifiers $\kappa_{V}$ and $\kappa_{F}$ for individual Higgs boson decay channels and their combination

\section{References}

1. ALEPH, DELPHI, L3, OPAL, SLD Collaborations, the LEP Electroweak Working Group, the SLD Electroweak and Heavy Flavour Working Groups. Precision electroweak measurements on the Z resonance. Phys. Rept. 427, 257 (2006). arXiv:hep-ex/0509008

2. ALEPH, DELPHI, L3, OPAL, the LEP Electroweak Working Group, Electroweak measurements in electron-positron collisions at W-boson-pair energies at LEP. Phys. Rept. 532, 119 (2013). arXiv: 1302.3415

3. D0 Collaboration, Observation of the top quark. Phys. Rev. Lett. 74, 2632 (1995). arXiv:hep-ex/9503003

4. CDF Collaboration, Observation of top quark production in $\bar{p} p$ collisions with the collider detector at Fermilab. Phys. Rev. Lett. 74, 2626 (1995). arXiv:hep-ex/9503002

5. The yearly reports of the LEP Electroweak Working Group can be found at http://lepewwg.web.cern.ch/LEPEWWG/stanmod

6. All versions of the PDG particle listings and reviews can be found at http://pdg.lbl.gov/2016/html/rpp_archives.html

7. Gfitter Group. Results at http://cern.ch/gfitter

8. HEPfit Group. Results at http://hepfit.roma1.infn.it

9. Gfitter Group, H. Flächer, M. Goebel, J. Haller, A. Hoecker, K. Mönig , J. Stelzer, Revisiting the global electroweak fit of the standard model and beyond with Gfitter. Eur. Phys. J. C 60, 543 (2009). arXiv:0811.0009. Erratum-ibid. C 71, 1718 (2011) 
10. ATLAS Collaboration, Observation of a new particle in the search for the standard model Higgs boson with the ATLAS detector at the LHC. Phys. Lett. B 716, 1 (2012). arXiv:1207.7214

11. CMS Collaboration, Observation of a new boson at a mass of 125 $\mathrm{GeV}$ with the CMS experiment at the LHC. Phys. Lett. B 716, 30 (2012). arXiv: 1207.7235

12. Yearly reports of the Tevatron Electroweak Working Group Top Subgroup can be found at https://tevewwg.fnal.gov/top

13. ATLAS, CDF, CMS, D0 Collaborations, First combination of Tevatron and LHC measurements of the top-quark mass. arXiv: 1403.4427

14. ATLAS Collaboration, Measurement of the top quark mass in the $t \bar{t} \rightarrow$ lepton+jets and $t \bar{t} \rightarrow$ dilepton channels using $\sqrt{s}=7 \mathrm{TeV}$ ATLAS data. Eur. Phys. J. C 75, 330 (2015). arXiv: 1503.05427

15. CMS Collaboration, Measurement of the top quark mass using proton-proton data at $\sqrt{s}=7$ and 8 TeV. Phys. Rev. D 93, 072004 (2016). arXiv:1509.04044

16. CDF, D0 Collaborations, Tevatron Electroweak Working Group, Combination of CDF and D0 results on the mass of the top quark using up $9.7 \mathrm{fb}^{-1}$ at the Tevatron. arXiv:1608.01881

17. D0 Collaboration, Combination of D0 measurements of the top quark mass. Phys. Rev. D 95, 112004 (2017). arXiv:1703.06994

18. ATLAS, CMS Collaborations, combined measurement of the Higgs boson mass in $p p$ collisions at $\sqrt{s}=7$ and $8 \mathrm{TeV}$ with the ATLAS and CMS experiments. Phys. Rev. Lett. 114, 191803 (2015). arXiv:1503.07589

19. ATLAS Collaboration, Measurement of the Higgs boson mass in the $H \rightarrow Z Z^{*} \rightarrow 4 \ell$ and $H \rightarrow \gamma \gamma$ channels with $\sqrt{s}=13 \mathrm{TeV}$ pp collisions using the ATLAS detector. Tech. Rep. ATLASCONF-2017-046 (2017)

20. CMS Collaboration, Measurements of properties of the Higgs boson decaying into the four-lepton final state in pp collisions at $\sqrt{s}=13$ TeV. JHEP 11, 047 (2017). arXiv:1706.09936

21. ATLAS Collaboration, Measurement of the top quark mass in the $t \bar{t} \rightarrow$ lepton+jets channel from $\sqrt{s}=8 \mathrm{TeV}$ ATLAS data, Tech. Rep. ATLAS-CONF-2017-071 (2017)

22. Gfitter Group, M, Baak et al., the electroweak fit of the standard model after the discovery of a new boson at the LHC. Eur. Phys. J. C 72, 2205 (2012). arXiv:1209.2716

23. Gfitter Group, M, Baak et al., The global electroweak fit at NNLO and prospects for the LHC and ILC. Eur. Phys. J. C 74, 3046 (2014). arXiv:1407.3792

24. ATLAS Collaboration, Measurement of the $W$-boson mass in pp collisions at $\sqrt{s}=7 \mathrm{TeV}$ with the ATLAS detector. Eur. Phys. J. C 78, 110 (2018). arXiv: 1701.07240

25. CDF, D0 Collaborations, Tevatron Run II combination of the effective leptonic electroweak mixing angle. arXiv:1801.06283

26. M. Davier, A. Hoecker, B. Malaescu, Z. Zhang, Reevaluation of the hadronic vacuum polarisation contributions to the Standard Model predictions of the muon $g-2$ and $\alpha\left(m_{Z}^{2}\right)$ using newest hadronic cross-section data. Eur. Phys. J. C 77, 827 (2017). arXiv: 1706.09436

27. H.E. Haber, G.L. Kane, T. Sterling, The fermion mass scale and possible effects of higgs bosons on experimental observables. Nucl. Phys. B 161, 493 (1979)

28. LEP, DELPHI, OPAL, ALEPH, L3 Collaborations, Search for charged higgs bosons: combined results using LEP data. Eur. Phys. J. C 73, 2463 (2013). arXiv:1301.6065

29. D0 Collaboration, Search for charged Higgs bosons decaying to top and bottom quarks in $p \bar{p}$ collisions. Phys. Rev. Lett. 102, 191802 (2009). arXiv:0807.0859

30. D0 Collaboration, Search for charged Higgs bosons in top quark decays. Phys. Lett. B 682, 278 (2009). arXiv:0908.1811

31. C.D.F. Collaboration, Search for charged Higgs bosons in decays of top quarks in $p \bar{p}$ collisions at $\sqrt{s}=1.96 \mathrm{TeV}$. Phys. Rev. Lett. 103, 101803 (2009). arXiv:0907.1269
32. ATLAS Collaboration, Search for a light charged Higgs boson in the decay channel $H^{+} \rightarrow c \bar{s}$ in $t \bar{t}$ events using pp collisions at $\sqrt{s}=7 \mathrm{TeV}$ with the ATLAS detector. Eur. Phys. J. C 73, 2465 (2013). arXiv:1302.3694

33. ATLAS Collaboration, Search for charged Higgs bosons decaying via $H^{ \pm} \rightarrow \tau^{ \pm} v$ in fully hadronic final states using $p p$ collision data at $\sqrt{s}=8 \mathrm{TeV}$ with the ATLAS detector. JHEP 03, 088 (2015). arXiv:1412.6663

34. ATLAS Collaboration, Search for charged Higgs bosons in the $H^{ \pm} \rightarrow t b$ decay channel in $p p$ collisions at $\sqrt{s}=8 \mathrm{TeV}$ using the ATLAS detector. JHEP 03, 127 (2016). arXiv:1512.03704

35. ATLAS Collaboration, Search for a Charged Higgs boson produced in the vector-boson fusion mode with decay $H^{ \pm} \rightarrow W^{ \pm} Z$ using $p p$ Collisions at $\sqrt{s}=8$ Tev with the ATLAS experiment. Phys. Rev. Lett. 114, 231801 (2015). arXiv:1503.04233

36. ATLAS Collaboration, Search for minimal supersymmetric standard model Higgs bosons $H / A$ and for a $Z^{\prime}$ boson in the $\tau \tau$ final state produced in $p p$ collisions at $\sqrt{s}=13 \mathrm{TeV}$ with the ATLAS detector. Eur. Phys. J. C 76, 585 (2016). arXiv:1608.00890

37. ATLAS Collaboration, Search for heavy $Z Z$ resonances in the $\ell^{+} \ell^{-} \ell^{+} \ell^{-}$and $\ell^{+} \ell^{-} \nu \bar{v}$ final states using proton-proton collisions at $\sqrt{s}=13 \mathrm{TeV}$ with the ATLAS detector. arXiv: 1712.06386

38. ATLAS Collaboration, Search for heavy resonances decaying into a $W$ or $Z$ boson and a Higgs boson in final states with leptons and $b$-jets in $36 \mathrm{fb}^{-1}$ of $\sqrt{s}=13 \mathrm{TeV} p p$ collisions with the ATLAS detector. arXiv: 1712.06518

39. ATLAS Collaboration, Search for additional heavy neutral Higgs and gauge bosons in the ditau final state produced in $36 \mathrm{fb}^{-1}$ of pp collisions at $\sqrt{s}=13 \mathrm{TeV}$ with the ATLAS detector. JHEP 01, 055 (2018). arXiv:1709.07242

40. ATLAS Collaboration, Search for heavy Higgs bosons $A / H$ decaying to a top quark pair in $p p$ collisions at $\sqrt{s}=8 \mathrm{TeV}$ with the ATLAS detector. Phys. Rev. Lett. 119, 191803 (2017). arXiv: 1707.06025

41. C.M.S. Collaboration, Search for a light charged Higgs boson in top quark decays in $p p$ collisions at $\sqrt{s}=7$ TeV. JHEP 07, 143 (2012). arXiv:1205.5736

42. CMS Collaboration, Search for a charged Higgs boson in pp collisions at $\sqrt{s}=8 \mathrm{TeV}$. JHEP 11, 018 (2015). arXiv: 1508.07774

43. CMS Collaboration, Search for a light charged Higgs boson decaying to $\mathrm{cs}$ in pp collisions at $\sqrt{s}=8 \mathrm{TeV}$. JHEP 12, 178 (2015). arXiv:1510.04252

44. CMS Collaboration, Search for neutral resonances decaying into a $\mathrm{Z}$ boson and a pair of b jets or $\tau$ leptons. Phys. Lett. B 759, 369 (2016). arXiv:1603.02991

45. Gfitter Group, M, Baak et al., Updated status of the global electroweak fit and constraints on new physics. Eur. Phys. J. C 72, 2003 (2012). arXiv:1107.0975

46. Particle Data Group, C. Patrignani et al., Review of Particle Physics. Chin. Phys. C 40, 100001 (2016)

47. ATLAS Collaboration, Measurement of the forward-backward asymmetry of electron and muon pair-production in $p p$ collisions at $\sqrt{s}=7 \mathrm{TeV}$ with the ATLAS detector. JHEP 09, 049 (2015). arXiv:1503.03709

48. CMS Collaboration, Measurement of the weak mixing angle with the forward-backward asymmetry of Drell-Yan events at $8 \mathrm{TeV}$. CMS Physics Analysis Summary CMS-PAS-SMP-16-007 (2017)

49. CDF, D0 Collaborations, Tevatron Electroweak Working Group, 2012 Update of the combination of CDF and D0 results for the mass of the W Boson. arXiv:1204.0042

50. ALEPH, DELPHI, L3, OPAL Collaborations, LEP Electroweak Working Group, Electroweak measurements in electron-positron collisions at W-boson-pair energies at LEP. Phys. Rept. 532, 119 (2013). arXiv: 1302.3415 
51. A. Valassi, Combining correlated measurements of several different physical quantities. Nucl. Instrum. Methods A 500, 391 (2003)

52. ATLAS Collaboration, Measurement of the top quark mass in the $t \bar{t} \rightarrow$ dilepton channel from $\sqrt{s}=8 \mathrm{TeV}$ ATLAS data. Phys. Lett. B 761, 350 (2016). arXiv:1606.02179

53. CMS Collaboration, Measurement of the $t \bar{t}$ production cross section and the top quark mass in the dilepton channel in $p p$ collisions at $\sqrt{s}=7$ TeV. JHEP 07, 049 (2011). arXiv:1105.5661

54. CMS Collaboration, Measurement of the top-quark mass in $t \bar{t}$ events with dilepton final states in $p p$ collisions at $\sqrt{s}=7 \mathrm{TeV}$. Eur. Phys. J. C 72, 2202 (2012). arXiv:1209.2393

55. CMS Collaboration, Measurement of the top-quark mass in $t \bar{t}$ events with lepton+jets final states in $p p$ collisions at $\sqrt{s}=7$ TeV. JHEP 12, 105 (2012). arXiv: 1209.2319

56. CMS Collaboration, Measurement of the top-quark mass in alljets $t \bar{t}$ events in pp collisions at $\sqrt{s}=7$ TeV. Eur. Phys. J. C 74, 2758 (2014). arXiv:1307.4617

57. CDF Collaboration, Final combination of the CDF results on topquark mass, Tech. Rep. CDF note 11080 (2014)

58. A.H. Hoang, A. Jain, I. Scimemi, I.W. Stewart, Infrared renormalization group flow for heavy quark masses. Phys. Rev. Lett. 101, 151602 (2008). arXiv:0803.4214

59. A.H. Hoang, I.W. Stewart, Top mass measurements from jets and the tevatron top-quark mass. Nucl. Phys. Proc. Suppl. 185, 220 (2008). arXiv:0808.0222

60. A. Buckley, J. Butterworth, S. Gieseke, D. Grellscheid, S. Hoeche et al., General-purpose event generators for LHC physics. Phys. Rept. 504, 145 (2011). arXiv:1101.2599

61. S. Moch, S. Weinzierl, S. Alekhin, J. Bluemlein, L. de la Cruz et al., High precision fundamental constants at the $\mathrm{TeV}$ scale. arXiv: 1405.4781

62. Mangano, M., private communication, Jun 2014. See also presentation at TOP2012 Interpreting the top quark mass: theoretical and MC aspects (2012)

63. P. Skands, D. Wicke, Non-perturbative QCD effects and the top mass at the tevatron. Eur. Phys. J. C 52, 133 (2007). arXiv:hep-ph/0703081

64. D. Wicke, P.Z. Skands, Non-perturbative QCD effects and the top mass at the tevatron. Nuovo Cim. B 123, S1 (2008). arXiv:hep-ph/0807.3248

65. P. Marquard, A.V. Smirnov, V.A. Smirnov, M. Steinhauser, Quark mass relations to four-loop order in perturbative QCD. Phys. Rev. Lett. 114, 142002 (2015). arXiv: 1502.01030

66. K. Chetyrkin, M. Steinhauser, The relation between the $\overline{\mathrm{MS}}$ and the on-shell quark mass at order $\alpha_{s}^{3}$. Nucl. Phys. B 573, 617 (2000). arXiv:hep-ph/9911434

67. K. Melnikov, T.v Ritbergen, The three-loop relation between the $\overline{\mathrm{MS}}$ and the pole quark masses. Phys. Lett. B 482, 99 (2000). arXiv:hep-ph/9912391

68. A. Hoang, M. Beneke, K. Melnikov, T. Nagano, A. Ota, Top - antitop pair production close to threshold: synopsis of recent NNLO results. Eur. Phys. J. Direct C 2, 1 (2000). arXiv:hep-ph/0001286

69. I. Dubovyk, A. Freitas, J. Gluza, T. Riemann, J. Usovitsch, The two-loop electroweak bosonic corrections to $\sin ^{2} \theta_{\mathrm{eff}}^{\mathrm{b}}$. Phys. Lett. B 762, 184 (2016). arXiv:1607.08375

70. M. Awramik, M. Czakon, A. Freitas, G. Weiglein, Compl fete two-loop electroweak fermionic corrections to $\sin ^{2} \theta_{\text {eff }}^{\text {lept }}$ and indirect determination of the Higgs boson mass. Phys. Rev. Lett. 93, 201805 (2004). arXiv:hep-ph/0407317

71. M. Awramik, M. Czakon, A. Freitas, Electroweak two-loop corrections to the effective weak mixing angle. JHEP 0611, 048 (2006). arXiv:hep-ph/0608099

72. L. Avdeev, J. Fleischer, S. Mikhailov, O. Tarasov, $\mathcal{O}\left(\alpha \alpha_{s}^{2}\right)$ correction to the electroweak $\rho$ parameter. Phys. Lett. B
336, 560. arXiv:hep-ph/9406363. Erratum-ibid. B 349, 597 (1994)

73. K. Chetyrkin, J.H. Kühn, M. Steinhauser, Corrections of order $\mathcal{O}\left(G_{F} M_{t}^{2} \alpha_{s}^{2}\right)$ to the $\rho$ parameter. Phys. Lett. B 351, 331 (1995). arXiv:hep-ph/9502291

74. K. Chetyrkin, J.H. Kühn, M. Steinhauser, QCD corrections from top quark to relations between electroweak parameters to order $\alpha_{s}^{2}$. Phys. Rev. Lett. 75, 3394 (1995). arXiv:hep-ph/9504413

75. J. van der Bij, K. Chetyrkin, M. Faisst, G. Jikia, T. Seidensticker, Three loop leading top mass contributions to the $\rho$ parameter. Phys. Lett. B 498, 156 (2001). arXiv:hep-ph/0011373

76. M. Faisst, J.H. Kühn, T. Seidensticker, O. Veretin, Three loop top quark contributions to the $\rho$ parameter. Nucl. Phys. B 665, 649 (2003). arXiv:hep-ph/0302275

77. Y. Schröder, M. Steinhauser, Four-loop singlet contribution to the $\rho$ parameter. Phys. Lett. B 622, 124 (2005). arXiv:hep-ph/0504055

78. K.G. Chetyrkin, M. Faisst, J.H. Kühn, P. Maierhofer, C. Sturm, Four-loop QCD corrections to the $\rho$ parameter. Phys. Rev. Lett. 97, 102003 (2006). arXiv:hep-ph/0605201

79. R. Boughezal, M. Czakon, Single scale tadpoles and $\mathcal{O}\left(G_{F} m_{t}^{2} \alpha_{s}^{3}\right)$ corrections to the $\rho$ parameter. Nucl. Phys. B 755, 221 (2006). arXiv:hep-ph/0606232

80. M. Awramik, M. Czakon, A. Freitas, B. Kniehl, Two-loop electroweak fermionic corrections to $\sin ^{2} \theta_{\mathrm{eff}}^{b \bar{b}}$. Nucl. Phys. B 813, 174 (2009). arXiv:0811.1364

81. M. Awramik, M. Czakon, A. Freitas, G. Weiglein, Precise prediction for the $\mathrm{W}$ boson mass in the standard model. Phys. Rev. D 69, 053006 (2004). arXiv:hep-ph/0311148

82. A. Freitas, Higher-order electroweak corrections to the partial widths and branching ratios of the $Z$ boson. JHEP 1404, 070 (2014). arXiv:1401.2447

83. A. Freitas, Two-loop fermionic electroweak corrections to the Zboson width and production rate. Phys. Lett. B 730, 50 (2014). arXiv: 1310.2256

84. A. Freitas, Y.-C. Huang, Electroweak two-loop corrections to $\sin ^{2} \theta_{\mathrm{eff}}^{\mathrm{bb}}$ and $R_{b}$ using numerical Mellin-Barnes integrals. JHEP 1208, 050 (2012). arXiv:1205.0299. Erratum-ibid. 1305 (2013) 074, Erratum-ibid. 1310 (2013) 044

85. K.G. Chetyrkin, J.H. Kühn, A. Kwiatkowski, QCD corrections to the $e^{+} e^{-}$cross-section and the $Z$ boson decay rate. Phys. Rept. 277, 189 (1996). arXiv:hep-ph/9503396

86. P. Baikov, K. Chetyrkin, J.H. Kühn, Order $\alpha_{s}^{4}$ QCD Corrections to $Z$ and $\tau$ Decays. Phys. Rev. Lett. 101, 012002 (2008). arXiv:0801.1821

87. P. Baikov, K. Chetyrkin, J.H. Kühn, J. Rittinger, Complete mathcal $O\left(\alpha_{s}^{4}\right)$ QCD corrections to hadronic Z-decays. Phys. Rev. Lett. 108, 222003 (2012). arXiv:1201.5804

88. A. Kataev, Higher order $\mathcal{O}\left(\alpha^{2}\right)$ and $\mathcal{O}\left(\alpha \alpha_{s}\right)$ corrections to $\sigma_{\text {total }}\left(e^{+} e^{-} \rightarrow\right.$ hadron $)$ and $Z$ boson decay rate. Phys. Lett. B 287, 209 (1992)

89. A. Czarnecki, J.H. Kühn, Nonfactorizable QCD and electroweak corrections to the hadronic $\mathrm{Z}$ boson decay rate. Phys. Rev. Lett. 77, 3955 (1996). arXiv:hep-ph/9608366

90. R. Harlander, T. Seidensticker, M. Steinhauser, Complete corrections of order $\alpha \alpha_{s}$ to the decay of the $\mathrm{Z}$ boson into bottom quarks. Phys. Lett. B 426, 125 (1998). arXiv:hep-ph/9712228

91. G.-C. Cho, K. Hagiwara, Y. Matsumoto, D. Nomura, The MSSM confronts the precision electroweak data and the muon $g-2$. JHEP 1111, 068 (2011). arXiv: 1104.1769

92. M.E. Peskin, T. Takeuchi, A New constraint on a strongly interacting Higgs sector. Phys. Rev. Lett. 65, 964 (1990)

93. M.E. Peskin, T. Takeuchi, Estimation of oblique electroweak corrections. Phys. Rev. D 46, 381 (1992) 
94. G.C. Branco, P.M. Ferreira, L. Lavoura, M.N. Rebelo, M. Sher, J.P. Silva, Theory and phenomenology of two-Higgs-doublet models. Phys. Rept. 516, 1 (2012). arXiv: 1106.0034

95. A. Arbey, F. Mahmoudi, O. Stål, T. Stefaniak, Status of the charged Higgs Boson in two higgs doublet models. arXiv: 1706.07414

96. D. Chowdhury, O. Eberhardt, Update of global two-higgs-doublet model fits. arXiv:1711.02095

97. ATLAS, CMS Collaborations, Measurements of the Higgs boson production and decay rates and constraints on its couplings from a combined ATLAS and CMS analysis of the LHC pp collision data at $\sqrt{s}=7$ and 8 TeV. JHEP 08, 045 (2016). arXiv:1606.02266

98. LHC Higgs Cross Section Working Group, J. R. Andersen et al., Handbook of LHC Higgs Cross Sections: 3. Higgs properties. arXiv: 1307.1347

99. D. Eriksson, J. Rathsman, O. Stål, 2HDMC: two-higgs-doublet model calculator physics and manual. Comput. Phys. Commun. 181, 189 (2010). arXiv:0902.0851

100. ATLAS Collaboration, Constraints on new phenomena via Higgs boson couplings and invisible decays with the ATLAS detector. JHEP 11, 206 (2015). arXiv: 1509.00672

101. Heavy Flavor Averaging Group (HFLAV), Y. Amhis et al., Averages of $b$-hadron, $c$-hadron, and $\tau$-lepton properties as of summer (2016). arXiv:1612.07233. Updated results at http://www. slac.stanford.edu/xorg/hflav

102. M. Misiak et al., Estimate of $B R\left(B \rightarrow X_{s} \gamma\right)$ at $\mathcal{O}\left(\alpha_{s}^{2}\right)$. Phys. Rev. Lett. 98, 022002 (2007). arXiv:hep-ph/0609232

103. M. Misiak et al., Updated NNLO QCD predictions for the weak radiative B-meson decays. Phys. Rev. Lett. 114, 221801 (2015). arXiv: 1503.01789

104. S. Aoki et al., Review of lattice results concerning low-energy particle physics. Eur. Phys. J. C 77, 112 (2017). arXiv:1607.00299

105. S. Fajfer, J.F. Kamenik, I. Nisandzic, On the $B \rightarrow D^{*} \tau \bar{\nu}_{\tau}$ sensitivity to new physics. Phys. Rev. D 85, 094025 (2012). arXiv: 1203.2654

106. LHCb, CMS Collaborations, Observation of the rare $B_{s}^{0} \rightarrow$ $\mu^{+} \mu^{-}$decay from the combined analysis of CMS and LHCb data. Nature 522, 68 (2015). arXiv:1411.4413

107. LHCb Collaboration, Measurement of the $B_{s}^{0} \rightarrow \mu^{+} \mu^{-}$branching fraction and effective lifetime and search for $B^{0} \rightarrow \mu^{+} \mu^{-}$ decays. Phys. Rev. Lett. 118, 191801 (2017). arXiv:1703.05747

108. BABAR Collaboration, Measurement of the $B \rightarrow X_{s} \gamma$ branching fraction and photon energy spectrum using the recoil method. Phys. Rev. D 77, 051103 (2008). arXiv:0711.4889

109. BABAR Collaboration, Precision measurement of the $B \rightarrow X_{s} \gamma$ photon energy spectrum, branching fraction, and direct $\mathrm{CP}$ asymmetry $A_{C P}\left(B \rightarrow X_{s+d} \gamma\right)$. Phys. Rev. Lett. 109, 191801 (2012). arXiv: 1207.2690

110. BABAR Collaboration, Exclusive measurements of $b \rightarrow s \gamma$ transition rate and photon energy spectrum. Phys. Rev. D 86, 052012 (2012). arXiv:1207.2520

111. Belle Collaboration, Measurement of inclusive radiative B-meson decays with a photon energy threshold of $1.7 \mathrm{GeV}$. Phys. Rev. Lett. 103, 241801 (2009). arXiv:0907.1384

112. Belle Collaboration, Measurement of the $\bar{B} \rightarrow X_{s} \gamma$ branching fraction with a sum of exclusive decays. Phys. Rev. D 91, 052004 (2015). arXiv:1411.7198

113. Belle Collaboration, Measurement of the inclusive $B \rightarrow X_{s+d} \gamma$ branching fraction, photon energy spectrum and HQE parameters. In Proceedings, 38th International Conference on High Energy Physics (ICHEP 2016): Chicago, IL, USA, August 3-10 (2016). arXiv: 1608.02344

114. CLEO Collaboration, Branching fraction and photon energy spectrum for $b \rightarrow s \gamma$. Phys. Rev. Lett. 87, 251807 (2001). arXiv:hep-ex/0108032
115. T. Hermann, M. Misiak, M. Steinhauser, $\bar{B} \rightarrow X_{s} \gamma$ in the Two higgs doublet model up to next-to-next-to-leading order in QCD. JHEP 11, 036 (2012). arXiv: 1208.2788

116. BABAR Collaboration, Evidence for an excess of $\bar{B} \rightarrow D^{(*)} \tau^{-} \bar{\nu}_{\tau}$ decays. Phys. Rev. Lett. 109, 101802 (2012). arXiv:1205.5442

117. BABAR Collaboration, Measurement of an excess of $\bar{B} \rightarrow$ $D^{(*)} \tau^{-} \bar{v}_{\tau}$ decays and implications for charged higgs bosons. Phys. Rev. D 88, 072012 (2013). arXiv:1303.0571

118. Belle Collaboration, Measurement of the branching ratio of $\bar{B} \rightarrow$ $D^{(*)} \tau^{-} \bar{v}_{\tau}$ relative to $\bar{B} \rightarrow D^{(*)} \ell^{-} \bar{v}_{\ell}$ decays with hadronic tagging at Belle. Phys. Rev. D 92, 072014 (2015). arXiv:1507.03233

119. Belle Collaboration, Measurement of the branching ratio of $\bar{B}^{0} \rightarrow D^{*+} \tau^{-} \bar{v}_{\tau}$ relative to $\bar{B}^{0} \rightarrow D^{*+} \ell^{-} \bar{v}_{\ell}$ decays with a semileptonic tagging method. Phys. Rev. D 94, 072007 (2016). arXiv: 1607.07923

120. Belle Collaboration, Measurement of the $\tau$ lepton polarization and $R\left(D^{*}\right)$ in the decay $\bar{B} \rightarrow D^{*} \tau^{-} \bar{\nu}_{\tau}$. Phys. Rev. Lett. 118, 211801 (2017). arXiv:1612.00529

121. LHCb Collaboration, Measurement of the ratio of branching fractions $\mathcal{B}\left(B^{O} \rightarrow D^{*+} \tau^{-} \mathbb{N}_{\tau}\right) / \mathcal{B}\left(\bar{B}^{0} \rightarrow D^{*+} \mu^{-} \bar{v}_{\mu}\right)$. Phys. Rev. Lett. 115, 111803 (2015). arXiv:1506.08614. Erratum-ibid. 115 (2015) 159901

122. T. Enomoto, R. Watanabe, Flavor constraints on the two Higgs doublet models of $\mathrm{Z}_{2}$ symmetric and aligned types. JHEP $\mathbf{0 5}, 002$ (2016). arXiv: 1511.05066

123. BABAR Collaboration, Evidence of $B^{+} \rightarrow \tau^{+} v$ decays with hadronic B tags. Phys. Rev. D 88, 031102 (2013). arXiv: 1207.0698

124. Belle Collaboration, Measurement of the branching fraction of $B^{+} \rightarrow \tau^{+} v_{\tau}$ decays with the semileptonic tagging method. Phys. Rev. D 92, 051102 (2015). arXiv: 1503.05613

125. G. Isidori, P. Paradisi, Hints of large $\tan \beta$ in flavour physics. Phys. Lett. B 639, 499 (2006). arXiv:hep-ph/0605012

126. X.-Q. Li, J. Lu, A. Pich, $B_{s, d}^{0} \rightarrow \ell^{+} \ell^{-}$decays in the aligned twohiggs-doublet model. JHEP 06, 022 (2014). arXiv: 1404.5865

127. X.-D. Cheng, Y.-D. Yang, X.-B. Yuan, Revisiting $B_{s} \rightarrow \mu^{+} \mu^{-}$ in the two-Higgs doublet models with $Z_{2}$ symmetry. Eur. Phys. J. C 76, 151 (2016). arXiv:1511.01829

128. T. Hermann, M. Misiak, M. Steinhauser, Three-loop QCD corrections to $B_{s} \rightarrow \mu^{+} \mu^{-}$. JHEP 12, 097 (2013). arXiv:1311.1347

129. C. Bobeth, M. Gorbahn, E. Stamou, Electroweak corrections to $B_{s, d} \rightarrow \ell^{+} \ell^{-}$. Phys. Rev. D 89, 034023 (2014). arXiv:1311.1348

130. C. Bobeth, M. Gorbahn, T. Hermann, M. Misiak, E. Stamou, M. Steinhauser, $B_{s, d} \rightarrow \ell^{+} \ell^{-}$in the standard model with reduced theoretical uncertainty. Phys. Rev. Lett. 112, 101801 (2014). arXiv: 1311.0903

131. BABAR Collaboration, Measurement of the absolute branching fractions for $D_{s}^{-} \rightarrow \ell^{-} \bar{\nu}_{\ell}$ and extraction of the decay constant $f_{D_{s}}$. Phys. Rev. D 82, 091103 (2010). arXiv:1008.4080. Erratum-ibid. D 91, 019901 (2015)

132. Belle Collaboration, Measurements of branching fractions of leptonic and hadronic $D_{s}^{+}$meson decays and extraction of the $D_{s}^{+}$ meson decay constant. JHEP 09, 139 (2013). arXiv:1307.6240

133. CLEO Collaboration, Improved measurement of absolute branching fraction of $D_{s}^{+} \rightarrow \tau^{+} v_{\tau}$. Phys. Rev. D 79, 052002 (2009). arXiv:0901.1147

134. CLEO Collaboration, Measurement of $\mathcal{B}\left(D_{s}^{+} \rightarrow \ell^{+} v\right)$ and the decay constant $f_{D^{+}}$from $600 \mathrm{pb}^{-1}$ of $e^{+} e^{-}$annihilation data near 4170 MeV. Phys. Rev. D 79, 052001 (2009). arXiv:0901.1216

135. CLEO Collaboration, Measurement of the pseudoscalar decay constant $f_{D_{s}}$ using $D_{s}^{+} \rightarrow \tau^{+} \nu, \tau^{+} \rightarrow \rho^{+} \bar{v}$ decays. Phys. Rev. D 80, 112004 (2009). arXiv:0910.3602

136. A.G. Akeroyd, F. Mahmoudi, Constraints on charged Higgs bosons from $D_{s}^{ \pm} \rightarrow \mu^{ \pm} v$ and $D_{s}^{ \pm} \rightarrow \tau^{ \pm} v$. JHEP 04, 121 (2009). arXiv:0902.2393 
137. Q. Chang, P.-F. Li, X.-Q. Li, $B_{s}^{0}-\bar{B}_{s}^{0}$ mixing within minimal flavor-violating two-Higgs-doublet models. Eur. Phys. J. C 75, 594 (2015). arXiv: 1505.03650

138. W.T. Ford, A. Lemonick, U. Nauenberg, P.A. Piroue, Comparison of the $K^{+}$and $K^{-}$decay rates into the $\tau$ and $K_{\mu 2}$ modes. Phys. Rev. Lett. 18, 1214 (1967)

139. CKMfitter Group, J. Charles et al., CP violation and the CKM matrix: assessing the impact of the asymmetric $B$ factories. Eur. Phys. J. C 41, 1 (2005). arXiv:hep-ph/0406184

140. CKMfitter Group, J. Charles et al., Current status of the standard model CKM fit and constraints on $\Delta F=2$ new physics. Phys. Rev. D 91, 073007 (2015). arXiv:1501.05013

141. CKMfitter Group, J. Charles et al., preliminary results as of Summer 2016 (ICHEP 2016 conference). http://ckmfitter.in2p3.fr/ www/results/plots_ichep16/ckm_res_ichep16.html

142. O. Deschamps et al., The two higgs doublet of type II facing flavour physics data. Phys. Rev. D 82, 073012 (2009). arXiv:0907.5135

143. Belle, BABAR Collaborations, the physics of the B factories. Eur. Phys. J. C 74, 3026 (2014). arXiv:1406.6311

144. FlaviaNet Working Group on Kaon Decays, M. Antonelli et al., An evaluation of $\left|V_{u s}\right|$ and precise tests of the standard model from world data on leptonic and semileptonic kaon decays. Eur. Phys. J. C 69, 399 (2010). arXiv:1005.2323

145. M. Misiak, M. Steinhauser, Weak radiative decays of the B meson and bounds on $M_{H^{ \pm}}$in the two-higgs-doublet model. Eur. Phys. J. C 77, 201 (2017). arXiv:1702.04571

146. M. Mühlleitner, M.O.P. Sampaio, R. Santos, J. Wittbrodt, Phenomenological comparison of models with extended higgs sectors. JHEP 08, 132 (2017). arXiv:1703.07750
147. P. Arnan, D. Beirevi, F. Mescia, O. Sumensari, Two Higgs doublet models and $b \rightarrow s$ exclusive decays. Eur. Phys. J. C 77, 796 (2017). arXiv:1703.03426

148. HPQCD Collaboration, H. Na, C. M. Bouchard, G. P. Lepage, C. Monahan and J. Shigemitsu, $B \rightarrow D \ell \nu$ form factors at nonzero recoil and extraction of $\left|V_{c b}\right|$. Phys. Rev. D 92, 054510 (2015). arXiv:1505.03925. Erratum-ibid. D 93 (2016) 119906

149. Muon G-2 Collaboration, G. W. Bennett et al., Final report of the muon E821 anomalous magnetic moment measurement at BNL. Phys. Rev. D 73, 072003 (2006). arXiv:hep-ex/0602035

150. A. Keshavarzi, D. Nomura, T. Teubner, The muon $g-2$ and $\alpha\left(M_{Z}^{2}\right)$ : a new data-based analysis. arXiv: 1802.02995

151. A. Broggio, E.J. Chun, M. Passera, K.M. Patel, S.K. Vempati, Limiting two-Higgs-doublet models. JHEP 11, 058 (2014). arXiv: 1409.3199

152. A. Cherchiglia, P. Kneschke, D. Stöckinger, H. Stöckinger-Kim, The muon magnetic moment in the 2HDM: complete two-loop result. JHEP 01, 007 (2017). arXiv: 1607.06292

153. H.E. Haber, Introductory low-energy supersymmetry. arXiv:hep-ph/9306207

154. H.E. Haber, H.E. Logan, Radiative corrections to the $Z b \bar{b}$ vertex and constraints on extended Higgs sectors. Phys. Rev. D 62, 015011 (2000). arXiv:hep-ph/9909335

155. C.D. Froggatt, R.G. Moorhouse, I.G. Knowles, Leading radiative corrections in two scalar doublet models. Phys. Rev. D 45, 2471 (1992) 